International Journal of Bifurcation and Chaos, Vol. 18, No. 1 (2008) 121-140

(c) World Scientific Publishing Company

\title{
COMPLEX INTERMITTENCY IN SWITCHING CONVERTERS
}

\author{
YUFEI ZHOU and JUN-NING CHEN \\ School of Electronic Science and Technology, \\ Anhui University, Hefei, Anhui, P. R. China \\ HERBERT H. C. IU \\ School of Electrical, Electronic and Computer Engineering, \\ The University of Western Australia, Australia \\ CHI K. TSE \\ Department of Electronic and Information Engineering, \\ Hong Kong Polytechnic University, Hong Kong, P. R. China \\ encktse@polyu.edu.hk
}

Received January 9, 2007; Revised January 29, 2007

\begin{abstract}
Intermittent instability is commonly observed in switching power supplies during the design and development phase. It manifests as symmetrical period-doubling bifurcation in the time domain with long intermittent periods. Such intermittent operation is considered undesirable in practice and is usually avoided by appropriate adjustments of circuit parameters. This paper explores the mechanism and conditions for the emergence of intermittency in a common voltage-mode controlled buck converter. It is found that interference at frequencies near the switching frequency or its rational multiples will induce intermittent operation. The strengths and frequencies of the interfering signals determine the type and period of intermittency. The problem is analyzed by transforming the conventional parameter-bifurcation analysis to a time-bifurcation analysis. Analytical results are verified by simulations and experimental measurements.
\end{abstract}

Keywords: Switching power converter; intermittency; bifurcation; chaos; nonlinear system.

\section{Introduction}

Intermittent operation, sometimes referred to as "breathing" in the physics literature $[\mathrm{Hu}$ et al., 2000; Qu et al., 1995; Yang et al., 1996], is a phenomenon which is frequently observed in periodically driven nonlinear systems. A specific form of intermittent operation exhibits period-doubling bifurcation in two symmetrical directions over the time domain. In this type of intermittent operation, the system intermittently bifurcates from the initial regular (or subharmonic) operation to the higher subharmonic operation or chaos, and then returns to the initial operation through the same bifurcation sequence in the reverse manner.
To distinguish it from the usual bifurcation in the parameter space, we refer to this intermittent bifurcation in the time domain as time-bifurcation, as it manifests as a change of qualitative behavior of the system as time elapses [Zhou et al., 2003] rather than as parameter changes. Such intermittency may arise in periodically driven nonlinear systems, where the frequency of a coupled signal is not consistent with the system's driving frequency. Switching power converters are periodically driven nonlinear systems, to which abundant sources of periodic interference are coupled via unintended paths (e.g. conducted or radiated paths) [Ferreira et al., 1997; William, 1994]. When the interference frequency 
differs from the switching frequency, and if the interference is strong enough, intermittent operation occurs [Tse et al., 2003]. Intermittent chaotic operation in a current-mode controlled boost converter has been studied recently [Wong et al., 2004]. In practice, intermittency is an undesirable operating state that should be avoided because frequent deviations from the intended working regime may increase device stresses and hence jeopardize reliability. As the emergence of intermittency in power supplies is a complex issue of practical importance, a through understanding of the mechanism and the conditions under which intermittency occurs is vital to the reliable design of power supplies.

This paper studies the simple voltage-mode controlled buck converter using a simple model that takes into account the coupling of intruding interference signal with the converter. With this model, the condition for the emergence of intermittency is studied in detail. Simulation and experimental results verify the analytical findings. In particular, we will identify the parameters that are crucial to the emergence of intermittency. We will show that when the frequency of the intruding interference is close to the switching frequency or its rational multiples, intermittency may emerge in a few different forms (or patterns). The traditional bifurcation analysis deals with bifurcation in a parameter space, and hence is not directly relevant to the present study. By "transferring" the analytical procedure from a parameter domain to the time domain, we consider the variation of the characteristic multipliers as time elapses and establish a few important findings regarding the appearance of intermittent bifurcation in the voltage-controlled buck converter. Computer simulation and experimental measurements verify the analytical results. This paper focuses on a voltagemode controlled buck converter, but the same modeling approach and analysis can be extended to other switching power converters as well as other periodically driven nonlinear systems.

\section{Voltage-Mode Controlled Buck Converter Coupled with Intruding Interference}

The buck converter consists of an inductor, a switch, a diode, a storage capacitor and a resistor load, which are connected as shown in Fig. 1(a). When switch $G$ turns on, the inductor current ramps up almost linearly, and when switch $G$ turns off, the inductor current ramps down and de-energizes through the diode to the load. In the voltage-mode control scheme, the output voltage error with respect to the reference voltage is amplified to give a control voltage $V_{\text {con }}$ :

$$
V_{\text {con }}=A\left(v_{o}-V_{\text {ref }}\right)
$$

which is then compared with a ramp signal $V_{\text {ramp }}$, defined as

$$
V_{\text {ramp }}=V_{L}+\left(V_{U}-V_{L}\right)\left(\frac{t}{T} \bmod 1\right),
$$

where all symbols are explained in Fig. 1. The comparator output, $u$, gives the pulse-width-modulated signal necessary for driving the switch. Typically, the switch $G$ is turned on when $V_{\text {con }} \leq V_{\text {ramp }}$, and turned off when $V_{\text {con }}>V_{\text {ramp }}$, as illustrated in Fig. 1(b). The state equations can be written as

$$
\begin{array}{ll}
\dot{x}=A_{\text {on }} x+B_{\text {on }} E & \text { for switch } G \text { on } \\
\dot{x}=A_{\text {off }} x+B_{\text {off }} E & \text { for switch } G \text { off }
\end{array}
$$

where $x$ denotes the state variables, i.e. $x=$ $\left[\begin{array}{ll}v_{o} & i_{L}\end{array}\right]^{T}$, the $A$ 's and $B$ 's are the system matrices given by

$$
\begin{gathered}
A_{\text {on }}=A_{\text {off }}=\left[\begin{array}{cc}
-\frac{1}{R C} & \frac{1}{C} \\
-\frac{1}{L} & 0
\end{array}\right], \\
B_{\text {on }}=\left[\begin{array}{l}
0 \\
\frac{1}{L}
\end{array}\right], \quad B_{\text {off }}=\left[\begin{array}{l}
0 \\
0
\end{array}\right] .
\end{gathered}
$$

When it operates under a common voltage-mode control, the buck converter has been shown to exhibit complex behavior [Banerjee \& Verghese, 2000; di Bernardo \& Vasca, 2000; Fossas \& Olivar, 1996; Hamill et al., 1992; Tse \& di Bernardo, 2002; Tse, 1994; Wong et al., 2004]. Figure 1(b) gives the key waveforms under this control. In practice, the intruding interference can take the form of coupling via conducted or radiated paths. Sometimes, the intruders can live on the same circuit board or be present at a very close proximity. Suppose the overall effect of the intruding signal is lumped to one spurious source $v_{s}$. We can model this coupling as an additive process which superposes the spurious signal $v_{s}$ directly on the control signal $V_{\text {con }}$, as shown in Fig. 1(a). The resulting perturbed control voltage is then given by

$$
V_{\text {con }}^{*}=V_{\text {con }}+v_{s}
$$

When the circuit parameters are chosen as follows: $L=20 \mathrm{mH}, C=47 \mu \mathrm{F}, R=22 \Omega$, 


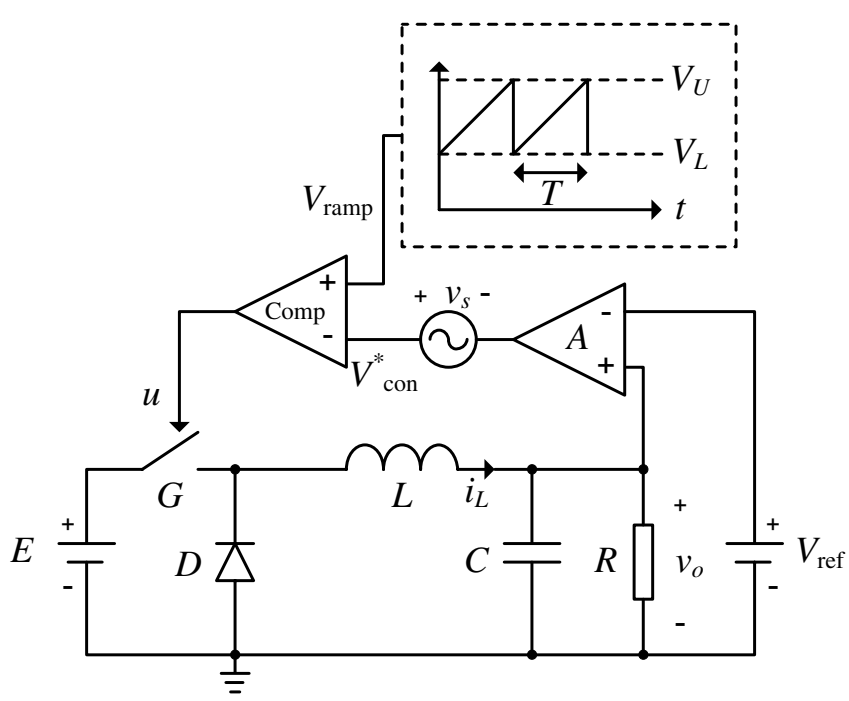

(a)

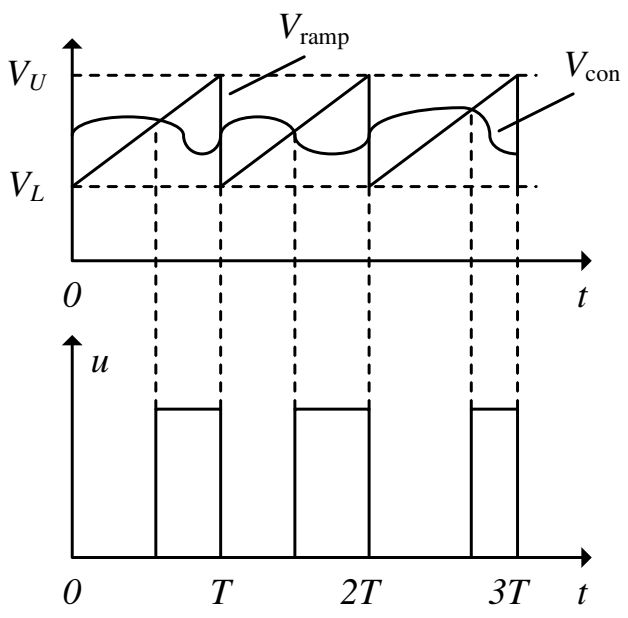

(b)

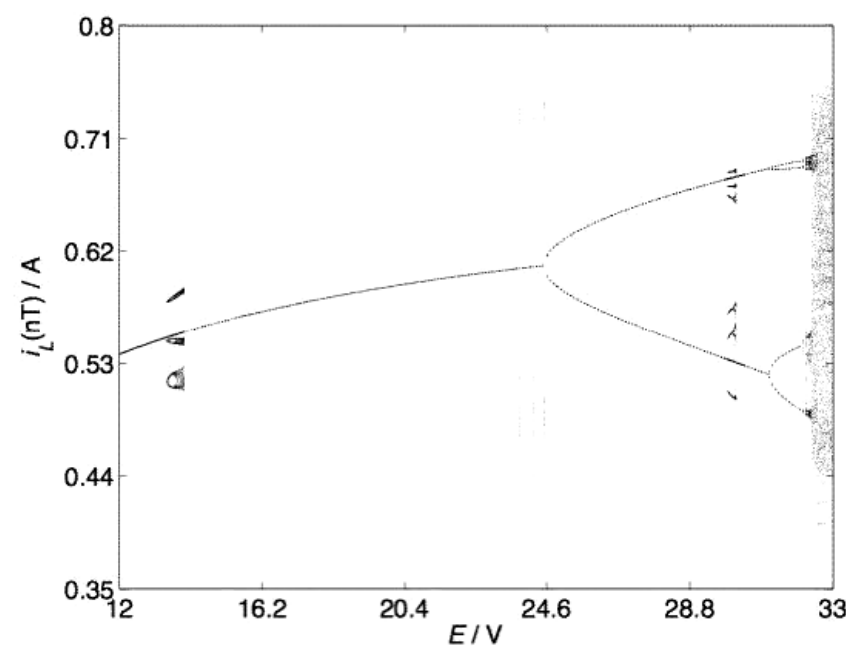

(c)

Fig. 1. Voltage-mode controlled buck converter coupled with spurious interference. (a) Schematic diagram; (b) key operation waveforms; (c) bifurcation diagram with $E$ as the bifurcation parameter.

$1 / T=f_{o}=2500 \mathrm{~Hz}, V_{\text {ref }}=11.3 \mathrm{~V}, A=8.4$, $V_{L}=3.8 \mathrm{~V}, V_{U}=8.2 \mathrm{~V}$, and without the interference $\left(v_{s}=0\right)$, the buck converter will experience a typical period-doubling bifurcation cascade with input voltage $E$ varying from $12 \mathrm{~V}$ to $33 \mathrm{~V}$ as shown in Fig. 1(c) [Fossas \& Olivar, 1996; Zhou et al., 2003]. The first bifurcation occurs when $E \approx 24.6 \mathrm{~V}$, and the buck converter eventually enters a chaotic region when $E \approx 32.3 \mathrm{~V}$.

\section{A Glimpse at the Phenomenon}

With the same set of circuit parameters shown above, the unperturbed buck converter operates in a regular period-1 orbit (steady state) when $E$ is fixed at $22 \mathrm{~V}$ [Fig. 2(a)], which corresponds to $V_{\text {con }} \approx 6.2 \mathrm{~V}$. In general, if we consider the interference $v_{s}$ being a periodic signal, e.g. sinusoidal signal with amplitude $\hat{v}_{s}$, the perturbed control voltage can be written as

$$
\begin{aligned}
V_{\text {con }}^{*} & =V_{\text {con }}+\hat{v}_{s} \sin \left(2 \pi f_{s} t\right) \\
& =V_{\text {con }}\left[1+\alpha_{v} \sin \left(2 \pi f_{s} t\right)\right]
\end{aligned}
$$

where $f_{s}$ is the frequency of the interference, and $\alpha_{v}$ is the strength of the interference which is defined as the ratio of the $\hat{v}_{s}$ to $V_{\text {con }}$, i.e. $\alpha_{v}=\hat{v}_{s} / V_{\text {con }}$. Here, we assume that in the steady state, the ripple of $V_{\text {con }}$ is negligible. Coupling with this intruding interference signal, the converter will operate in a range of 


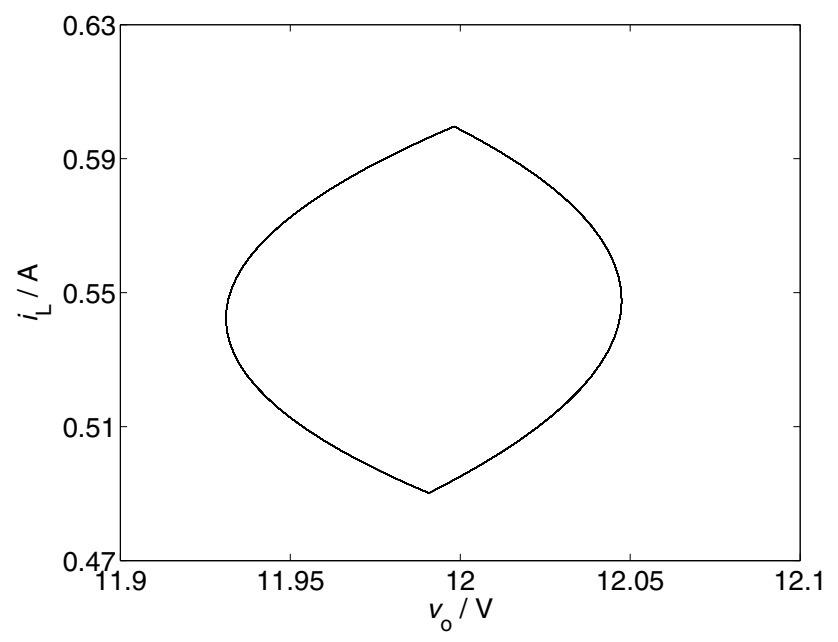

(a)

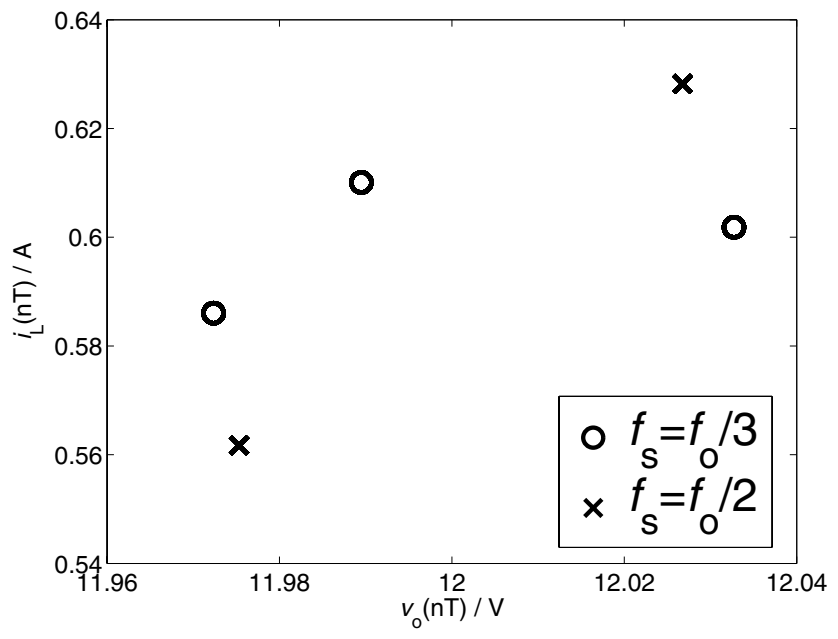

(c)

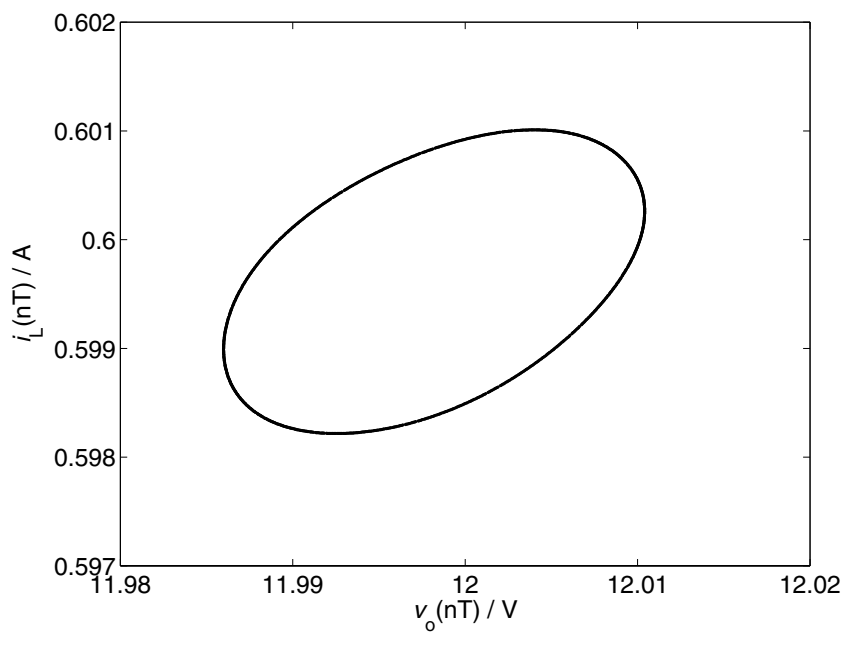

(b)

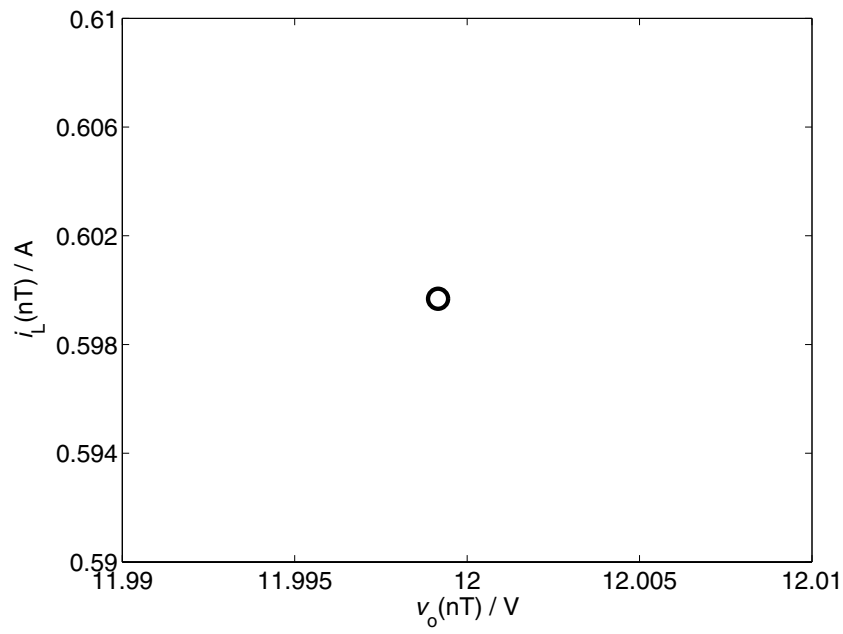

(d)

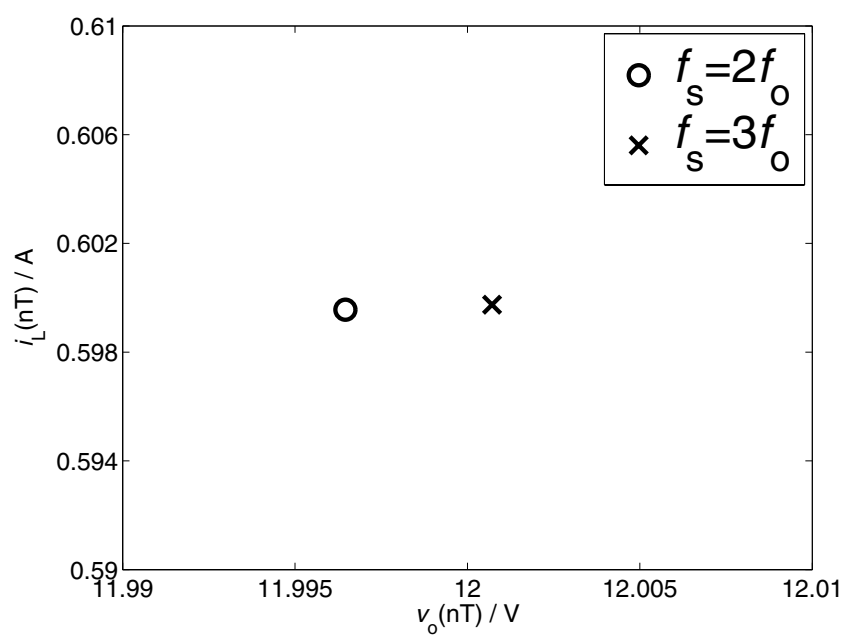

(e)

Fig. 2. (a) Period-1 operation; Poincaré sections of responses induced by different levels of interference with (b) $\alpha_{f}=\sqrt{3} / 2$, $\alpha_{v}=0.015 ;$ (c) $\alpha_{f}=1 / 2$ or $1 / 3, \alpha_{v}=0.021$; (d) $\alpha_{f}=1, \alpha_{v}=0.0046$; (e) $\alpha_{f}=2$ or $3, \alpha_{v}=0.0046$. 
possible regimes, such as periodic operation, quasiperiodic operation, etc., according to the frequency ratio $\alpha_{f}\left(\alpha_{f}=f_{s} / f_{o}\right)$. We can summarize them in two cases.

\section{Case 1. Frequency ratio $\alpha_{f}$ is an irrational number}

In this case, the buck converter exhibits quasiperiodic behavior, because there are two incommensurate frequencies, i.e. switching frequency $f_{o}$ and interference frequency $f_{s}$ in the system. The quasi-periodicity is characterized by a torus on the Poincaré section. Figure 2(b) shows an example with $\alpha_{f}=\sqrt{3} / 2$ and $\alpha_{v}=0.015$.

\section{Case 2. Frequency ratio $\alpha_{f}$ is a rational number}

If $\alpha_{f}$ is a rational number, we have

$$
\alpha_{f}=\frac{f_{s}}{f_{o}}=\frac{N_{\text {num }}}{N_{\text {den }}}
$$

where $N_{\text {num }}$ and $N_{\text {den }}$ are positive integers. In this case, the buck converter operates periodically with period number equalling $N_{\text {den }}$, i.e. have an operation of period- $N_{\text {den }}$ subharmonics. We can further summarize them in two subcases.

(a) $\alpha_{f}=1 / N_{\text {den }}$ : In this subcase, the buck converter operates in a period- $N_{\text {den }}$ subharmonic orbit. Figures 2(c) and 2(d) show three examples with $N_{\text {den }}=3,2$, 1 , which correspond to period-3, period-2 and period-1 operation, respectively, and are characterized by 3,2 and 1 intersections on the Poincaré section.

(b) $\alpha_{f}=N_{\text {num }}$ : In this subcase, the buck converter operates in a period-1 orbit definitely. Figures 2(d) and 2(e) show three examples with $N_{\text {num }}=1,2,3$, all corresponding to period-1 operation, and being characterized by one intersection on the Poincaré section.

\section{Intermittency: A Detailed Look at the Time-Bifurcation Diagrams}

Switching power converters are susceptible to abundant sources of periodic interference via unintended paths (e.g. conducted or radiated paths) [Ferreira et al., 1997; William, 1994]. As the intruding signal is coupled unintentionally, its frequency $f_{s}$ is usually not equal to the switching frequency $f_{o}$, or its rational multiples exactly. Therefore, it is unlikely to observe the kind of operation given in Fig. 2. But it is possible that the interference frequency $f_{s}$ approaches the switching frequency $f_{o}$, or its rational multiples. In this situation, we have $f_{s}=n f_{o}+\hat{f}$, i.e. $\alpha_{f}=n+\hat{f} / f_{o}$, where $\hat{f}$ is a small number compared to $f_{o}$ and $n$ is a rational number. This kind of interference will induce intermittency in a few different forms (such as intermittent subharmonics, intermittent chaos). The type and period of intermittency are determined by the interference frequency $f_{s}$ and strength $\alpha_{v}$.

When the interference frequency is close to the switching frequency, or its rational multiples, the perturbed control voltage (7) can be rewritten as

$$
\begin{aligned}
V_{\text {con }}^{*} & =V_{\text {con }}\left[1+\alpha_{v} \sin \left(2 \pi f_{s} t\right)\right] \\
& =V_{\text {con }}\left[1+\alpha_{v} \sin 2 \pi\left(n f_{o}+\hat{f}\right) t\right] .
\end{aligned}
$$

Assuming $\hat{f}=1$, and $n$ being $1 / 2,1$ and 2, respectively, we can obtain the time-bifurcation diagrams of intermittency, as shown in Figs. 3 to 5. From these figures, we have the following observations.

(1) When the strength of the interference is very weak (i.e. small $\alpha_{v}$ ), the converter can still maintain its expected steady-state period- $N_{\text {den }}$ operation, though the average operating point may fluctuate. The effect of the interference is not significant at this stage, and no intermittency shows up. Figures 3(a), 4(a) and 5(a) show the corresponding time-bifurcation diagrams.

(2) As the interference signal strength increases, the converter experiences higher subharmonic operation intermittently on top of the period$N_{\text {den }}$ operation. For a relatively low interference signal strength, period- $2 N_{\text {den }}$ subharmonics are observed intermittently with period- $N_{\text {den }}$. Figures 3(b), 4(b) and 5(b) give the corresponding time-bifurcation diagrams.

(3) Further increase in interference signal strength causes period- $2^{2} N_{\text {den }}$ subharmonics to occur intermittently with period- $2 N_{\text {den }}$ subharmonics and the period- $N_{\text {den }}$ operation, as shown in Figs. 3(c), 4(c) and 5(c).

(4) For a sufficiently high intruding signal strength, the converter starts to experience chaotic operation intermittently with period- $2^{i} N_{\text {den }}$ subharmonics and the period- $N_{\text {den }}$ operation, as shown in Figs. 3(d), 4(d) and 5(d).

(5) The intermittent period $T_{\mathrm{int}}$ is represented as

$$
T_{\mathrm{int}}=\frac{1}{N_{\mathrm{den}} \hat{f}}
$$

where $\hat{f}=\left|f_{s}-n f_{o}\right|$. Thus, if the interference signal frequency is very close to the switching 


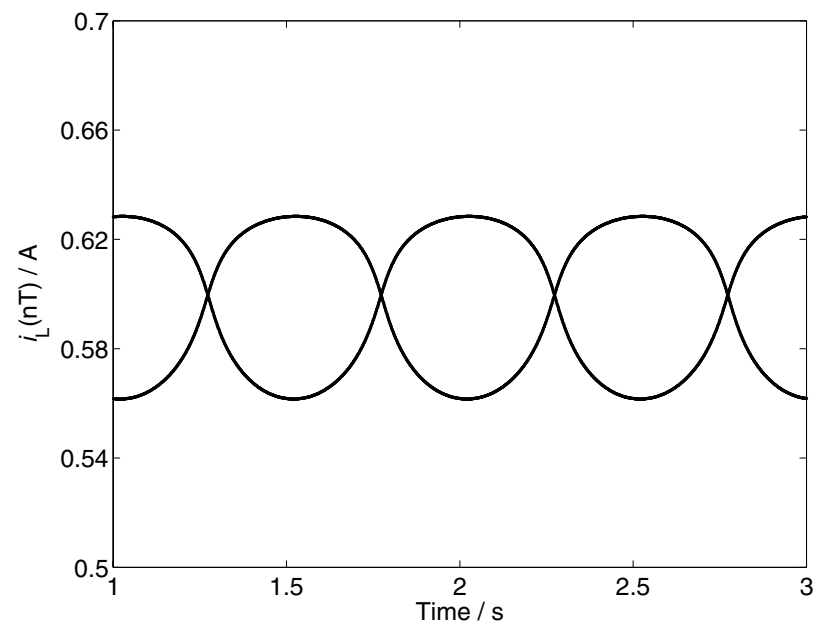

(a)

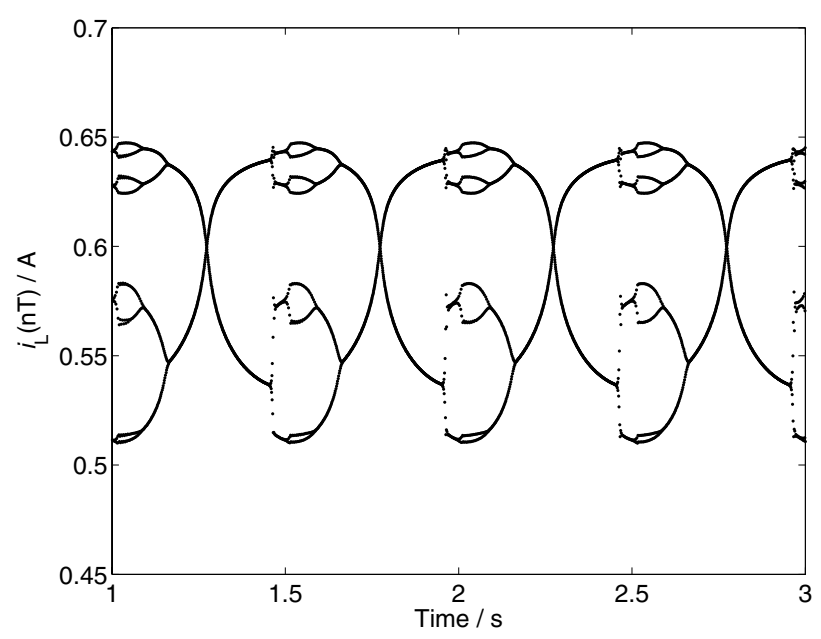

(c)

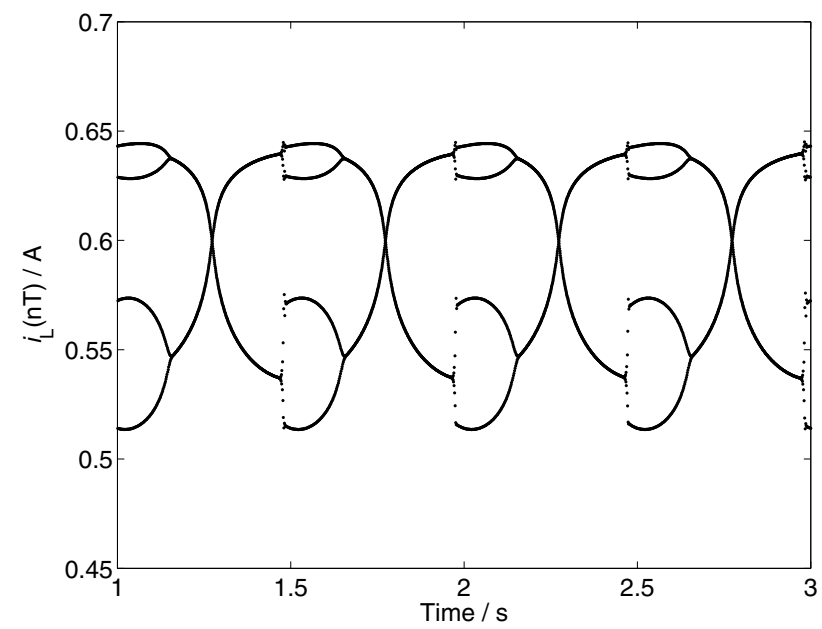

(b)

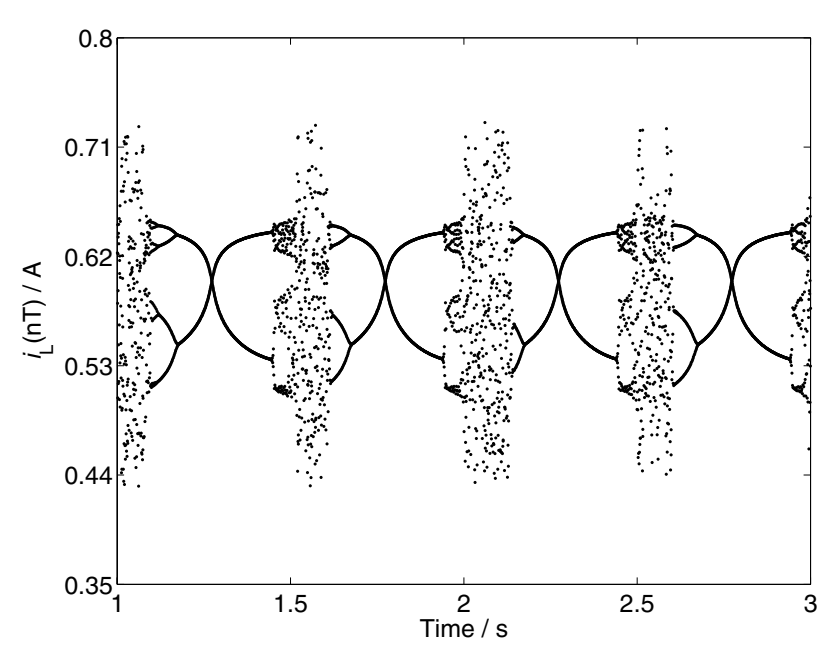

(d)

Fig. 3. Sampled inductor current waveforms (time-bifurcation diagrams) for $f_{s}=n f_{o}+\hat{f}=\frac{1}{2} f_{o}+1=1251 \mathrm{~Hz}$. (a) $\alpha_{v}=0.021$; (b) $\alpha_{v}=0.066 ;$ (c) $\alpha_{v}=0.069 ;$ (d) $\alpha_{v}=0.075$.

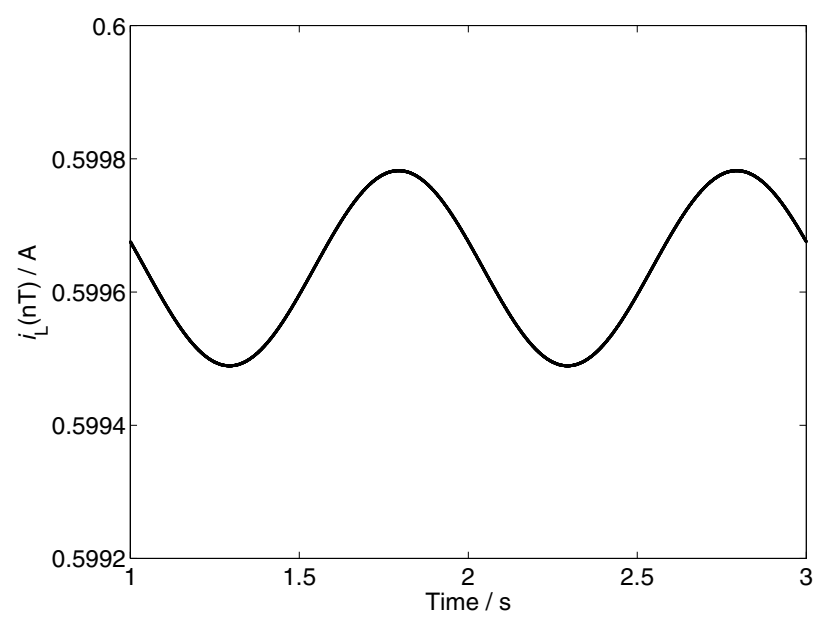

(a)

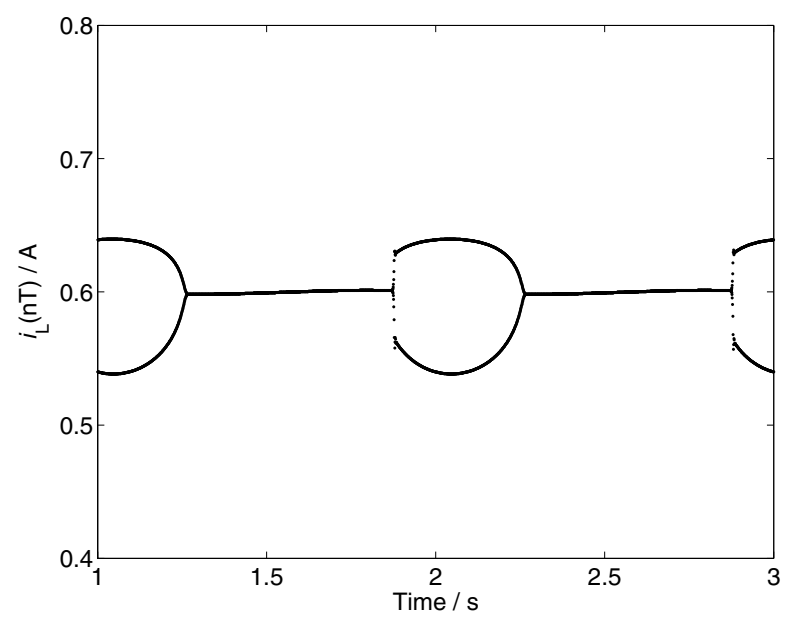

(b)

Fig. 4. Sampled inductor current waveforms (time-bifurcation diagram) for $f_{s}=n f_{o}+\hat{f}=f_{o}+1=2501 \mathrm{~Hz}$. (a) $\alpha_{v}=0.0046$; (b) $\alpha_{v}=0.046 ;$ (c) $\alpha_{v}=0.054 ;$ (d) $\alpha_{v}=0.069$. 


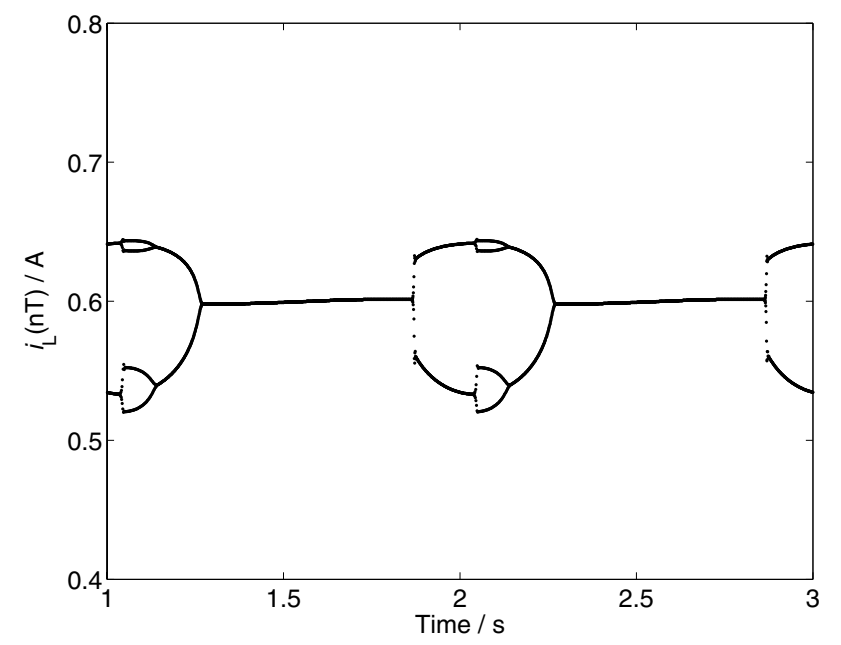

(c)

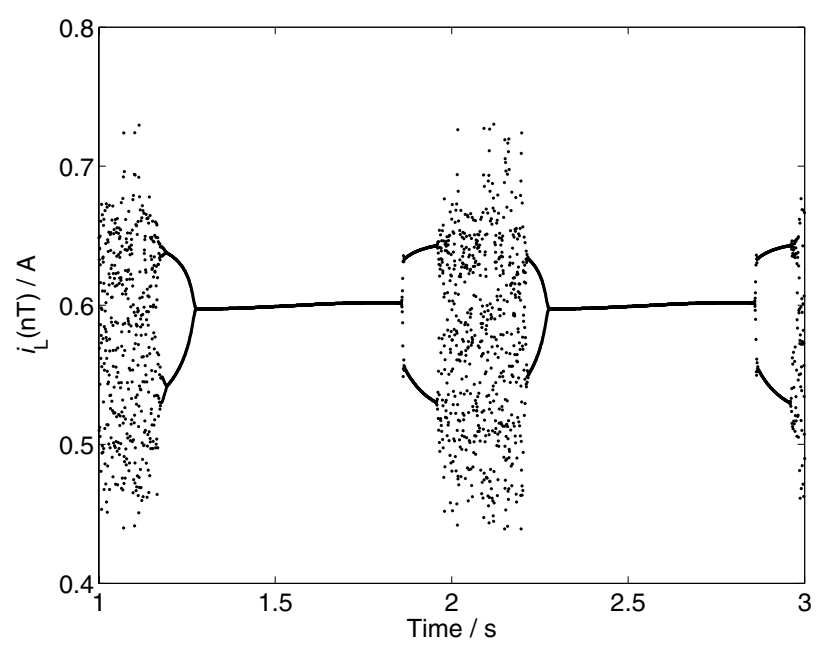

(d)

Fig. 4. (Continued)

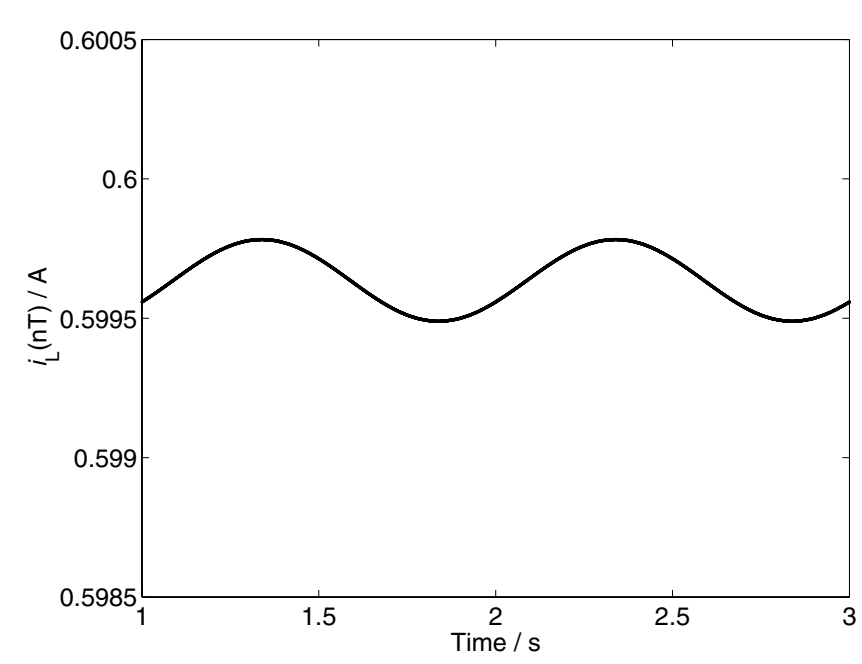

(a)

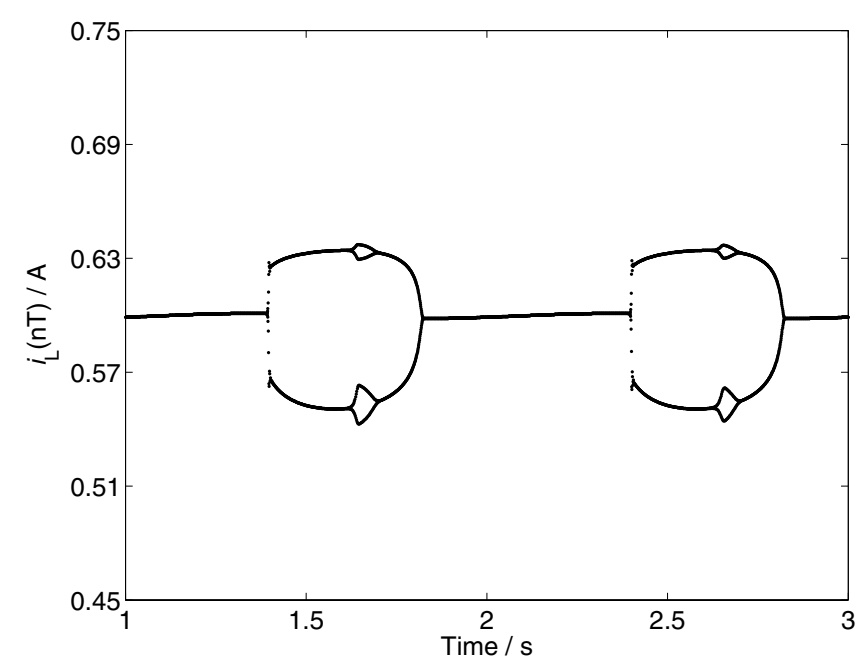

(c)

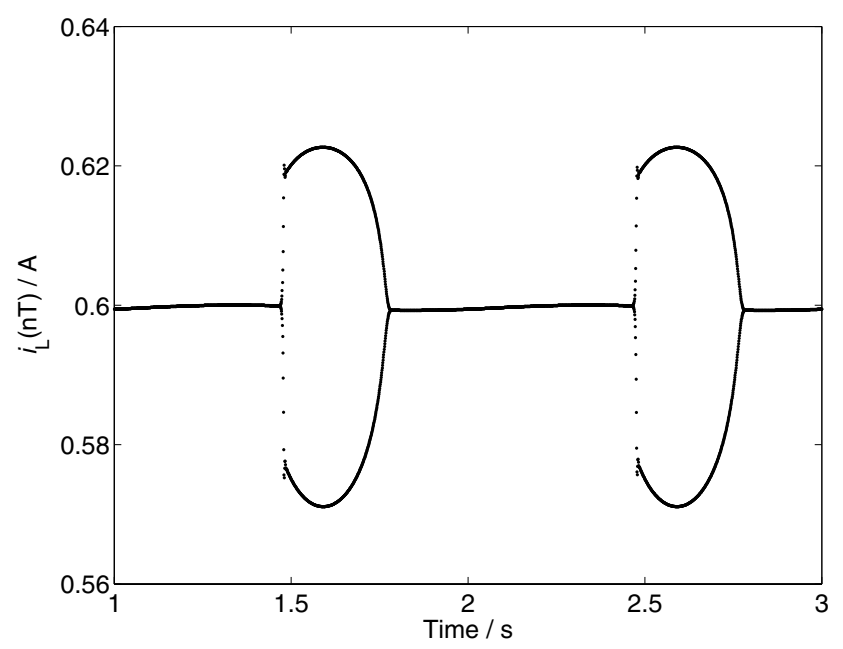

(b)

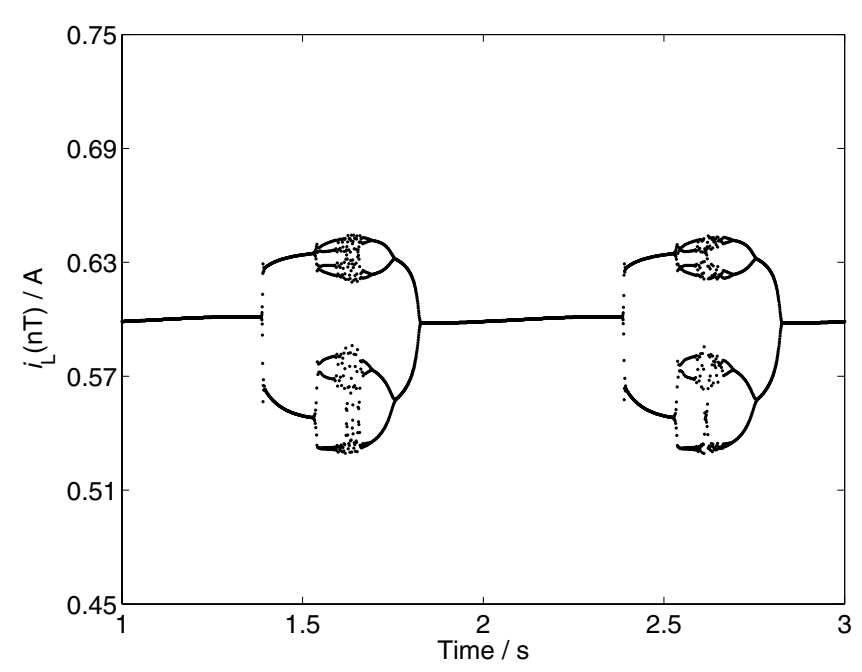

(d)

Fig. 5. Sampled inductor current waveforms (time-bifurcation diagrams) for $f_{s}=n f_{o}+\hat{f}=2 f_{o}+1=5001 \mathrm{~Hz}$. (a) $\alpha_{v}=$ $0.0046 ;$ (b) $\alpha_{v}=0.012 ;$ (c) $\alpha_{v}=0.041 ;$ (d) $\alpha_{v}=0.053$. 
frequency of the converter, or its rational multiples, the intermittency is long. In the examples here, $T_{\text {int }}=1 \mathrm{~s}$ with $\hat{f}=1 \mathrm{~Hz}$ for the case of $N_{\text {den }}=1$, which are shown in Figs. 4 and 5 ; and $T_{\text {int }}=1 / 2 \mathrm{~s}$ with $\hat{f}=1 \mathrm{~Hz}$ for the case of $N_{\text {den }}=2$, which are shown in Fig. 3 .

\section{Experimental Observations}

We have built a circuit prototype to verify the simulation results. The circuit parameters are same as those used in the simulations. Without any interference, the converter will operate in the original period-1 orbit as shown in Fig. 6 for $E=22 \mathrm{~V}$. Sinusoidal intruding interference signals have been used in the experiment. For consistency, here we provide three typical cases which correspond to the simulations reported in the last section, i.e. $n$ equals $1 / 2,1$ and 2 . These cases will also be analyzed in Secs. 6 and 7. By varying the interference signal strength, we observe intermittent subharmonics and chaos, which are consistent with the simulations. Figures 7 to 9 show a few time-bifurcation diagrams obtained experimentally, in which upper traces are output voltage $v_{o}$ and lower traces are inductor current $i_{L}$.

(1) Figure 7 shows the case when the interference frequency nears $f_{o} / 2$. As the interference strength $\alpha_{v}$ increases, we observe fluctuating period-2 operation (Fig. 7(a), $v_{o}: 200 \mathrm{mV} /$ div, $i_{L}: 100 \mathrm{~mA} /$ div), intermittent subharmonic operation (Fig. 7(b), $v_{o}: 200 \mathrm{mV} /$ div, $i_{L}$ : $100 \mathrm{~mA} / \mathrm{div}$ ) and chaos (Fig. 7(c), $v_{o}$ : $\left.500 \mathrm{mV} / \operatorname{div}, i_{L}: 250 \mathrm{~mA} / \operatorname{div}\right)$.

(2) Figure 8 represents the case when the interference frequency is close to $f_{o}$. As the interference

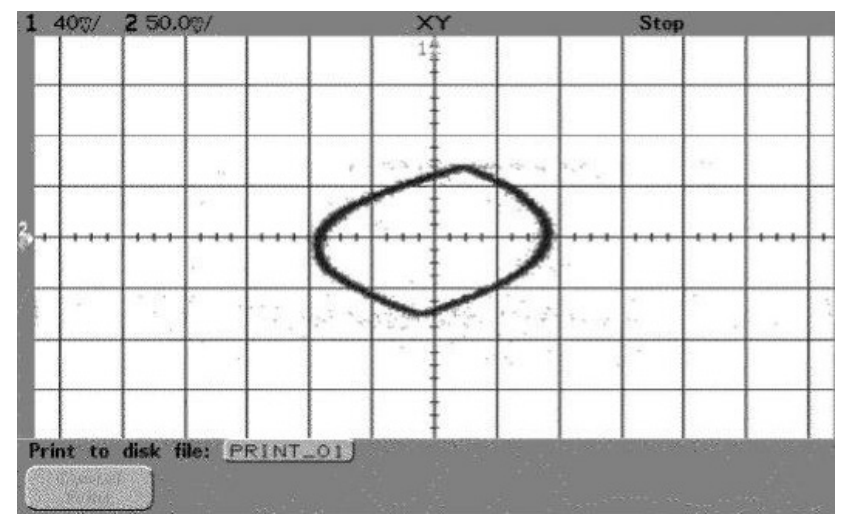

Fig. 6. Measured phase portrait of period-1 operation with $E=22 \mathrm{~V}, x$-axis: output voltage $v_{o}, 40 \mathrm{mV} / \operatorname{div} ; y$-axis: inductor current $i_{L}, 50 \mathrm{~mA} /$ div.

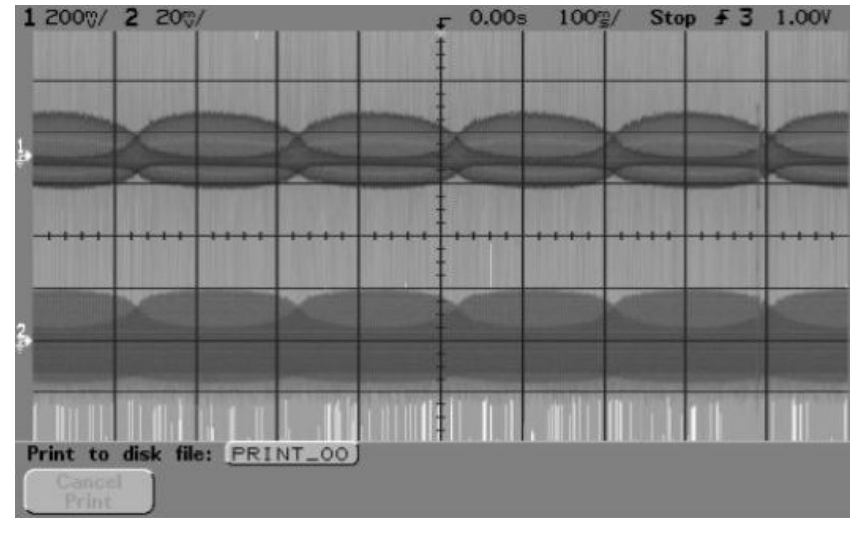

(a)

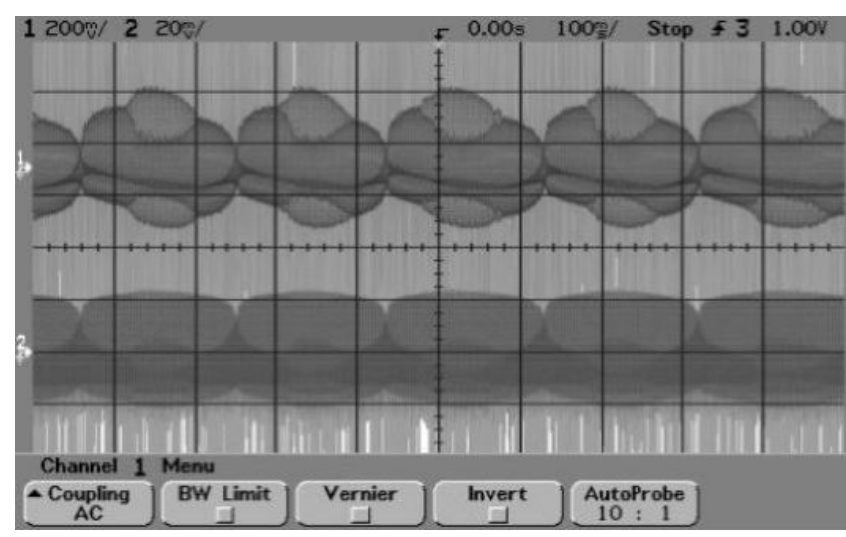

(b)

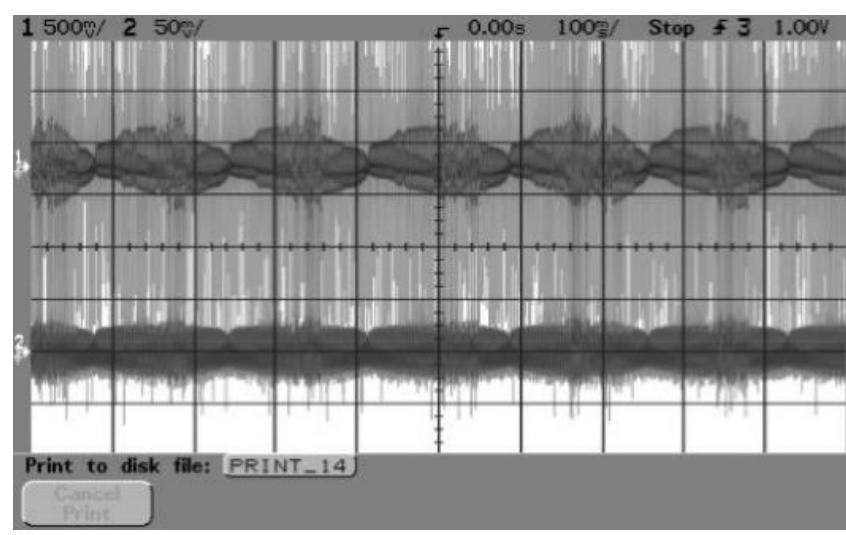

(c)

Fig. 7. Measured time-bifurcation diagrams with sinusoidal interference of different strengths $\alpha_{v}$ and $n=1 / 2$. (a) $\alpha_{v}=$ 0.021 ; (b) $\alpha_{v}=0.067$; (c) $\alpha_{v}=0.093$.

strength increases, we observe intermittent subharmonics (Fig. 8(a), $v_{o}: 90 \mathrm{mV} / \mathrm{div}, i_{L}$ : $100 \mathrm{~mA} /$ div and Fig. 8(b), $v_{o}: 150 \mathrm{mV} /$ div, $i_{L}: 100 \mathrm{~mA} / \mathrm{div}$ ) and chaos (Fig. 8(c), $v_{o}$ : $250 \mathrm{mV} /$ div, $i_{L}: 200 \mathrm{~mA} /$ div). Here, the fluctuating period-1 operation cannot be observed, 


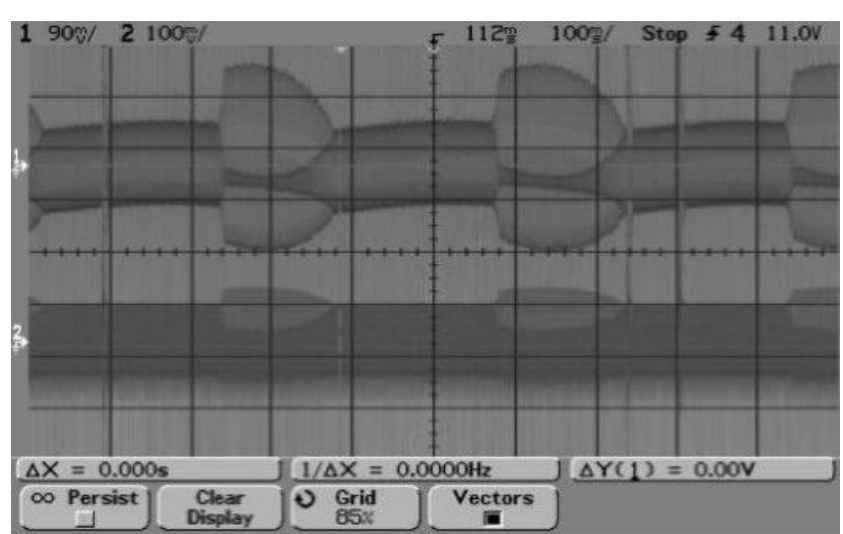

(a)

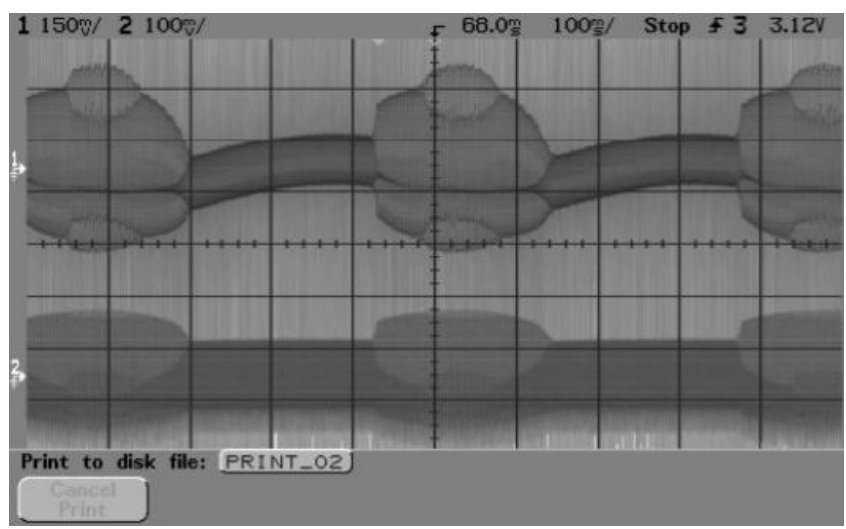

(b)

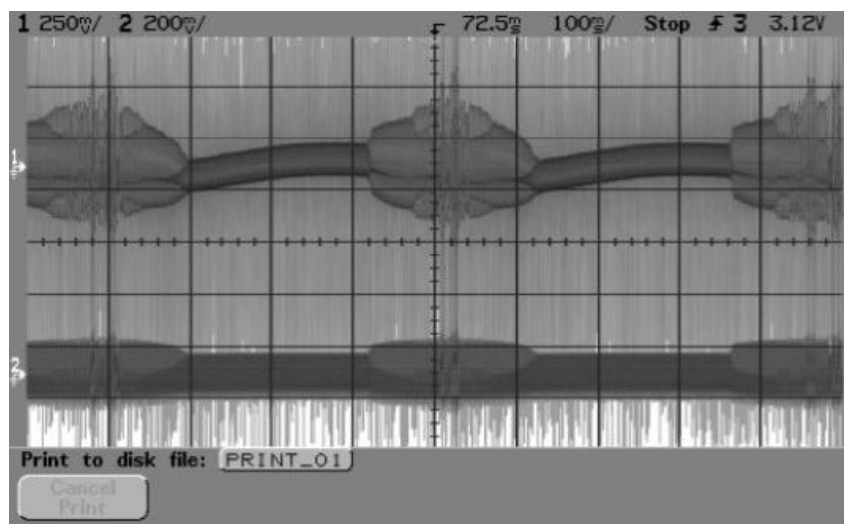

(c)

Fig. 8. Measured time-bifurcation diagrams with sinusoidal interference of different strengths $\alpha_{v}$ and $n=1$. (a) $\alpha_{v}=$ 0.015 ; (b) $\alpha_{v}=0.061$; (c) $\alpha_{v}=0.064$.

because the minimum output of the signal generator used here will still induce the intermittent subharmonics.

(3) Figure 9 shows the case when the interference frequency is close to $2 f_{o}$. Similar to the case in Fig. 8, as the interference strength increases, we

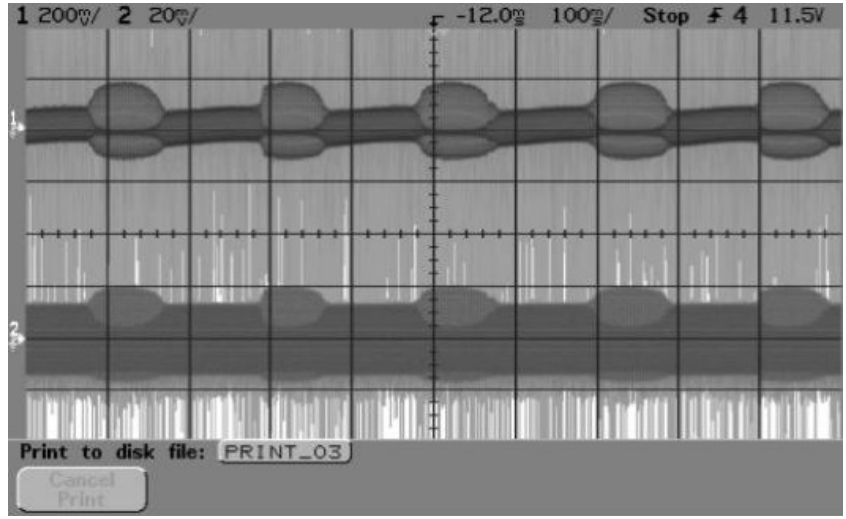

(a)

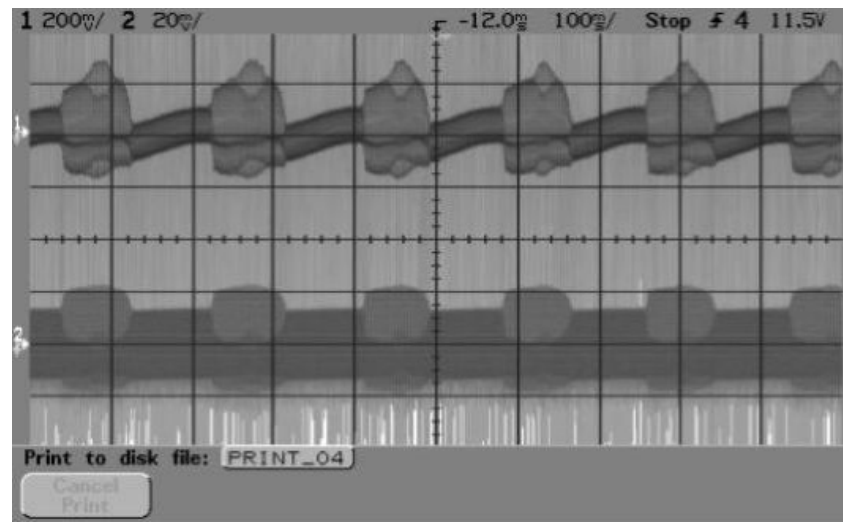

(b)

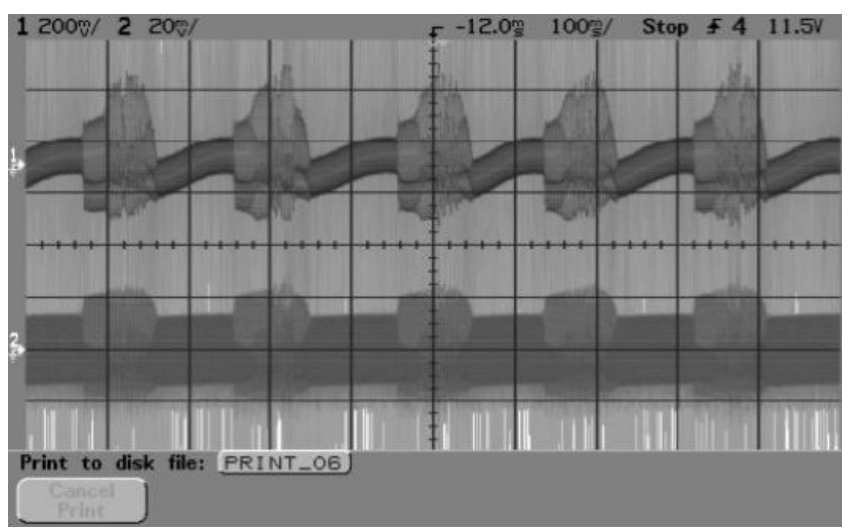

(c)

Fig. 9. Measured time-bifurcation diagrams with sinusoidal interference of different strengths $\alpha_{v}$ and $n=2$. (a) $\alpha_{v}=$ 0.012 ; (b) $\alpha_{v}=0.052$; (c) $\alpha_{v}=0.061$.

observe intermittent subharmonics (Figs. 9(a) and $9(\mathrm{~b}), v_{o}: 200 \mathrm{mV} / \mathrm{div}, i_{L}: 100 \mathrm{~mA} /$ div $)$ and chaos (Fig. 9(c), $v_{o}: 200 \mathrm{mV} / \operatorname{div}, i_{L}$ : $100 \mathrm{~mA} /$ div). The fluctuating period-1 operation is absent for the same reason as stated previously. 


\section{Mapping from Time-Bifurcation to Parameter-Bifurcation}

As the traditional bifurcation analysis mainly deals with parameter-bifurcations, it is not directly applicable to the analysis of intermittent bifurcation in the present study. Before we analyze the observed intermittency, we need to apply a transformation, which will transfer the change of time to the change of another variable. As we will see, the new variable is a conceptual "phase shift", which is used to represent the equivalent drift in the interfering frequency from the switching frequency. Basically we express the perturbed control voltage, originally

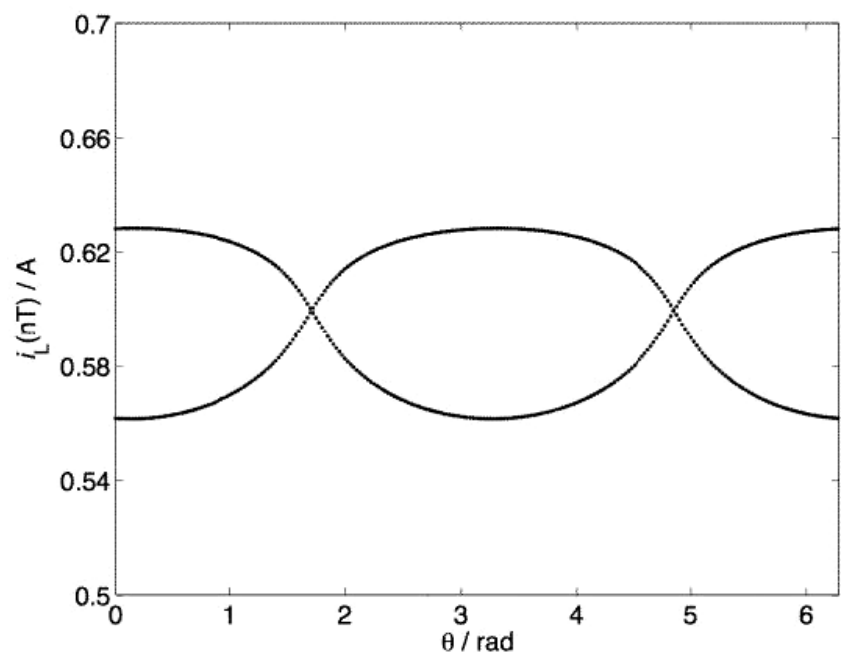

(a)

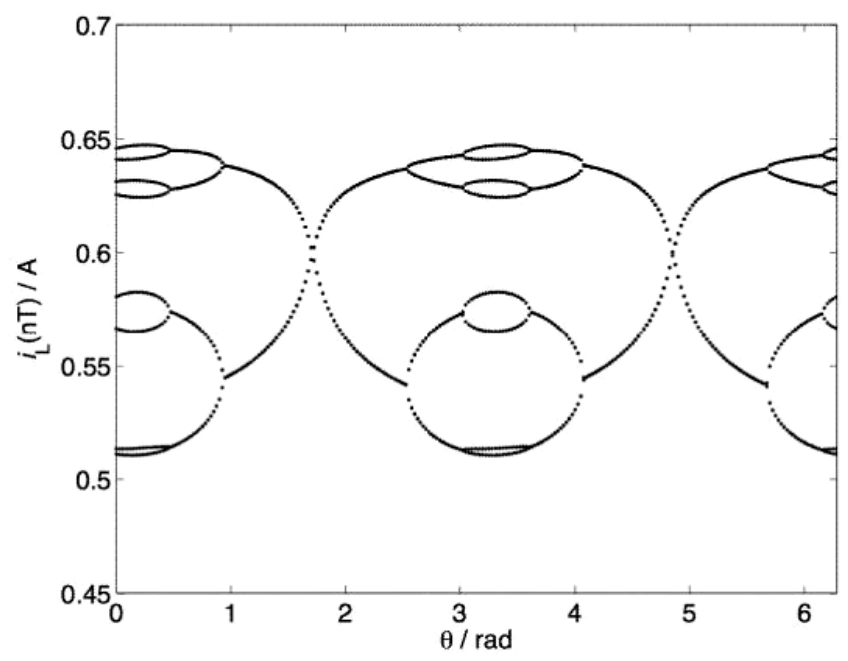

(c) given in (9), as

$$
\begin{aligned}
V_{\text {con }}^{*} & =V_{\text {con }}\left\{1+\alpha_{v} \sin \left[2 \pi\left(n f_{o}+\hat{f}\right) t\right]\right\} \\
& =V_{\text {con }}\left[1+\alpha_{v} \sin \left(2 \pi n f_{o} t+\theta\right)\right]
\end{aligned}
$$

where $\theta=2 \pi \hat{f} t$. After this transformation, a sinusoidal term with the phase shift $\theta$, and at the same frequency as the switching frequency $f_{o}$ or its rational multiples, is obtained. Now we can map the change of the time over interval $[0,1 / \hat{f}]$ onto the change of phase shift $\theta$ over interval $[0,2 \pi]$.

Using the above perturbed control voltage, a parameter-bifurcation diagram can be constructed by selecting $\theta$ as the bifurcation parameter, which

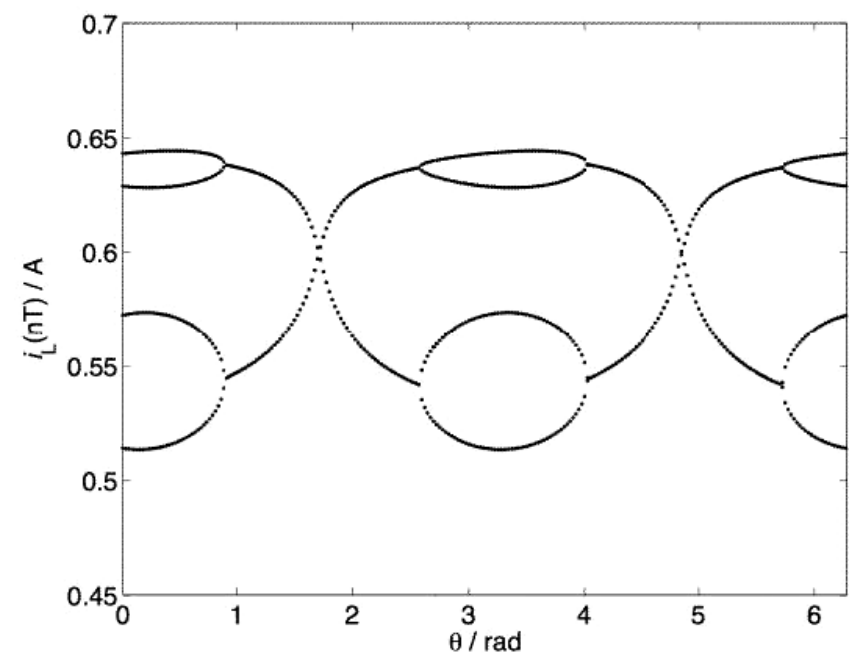

(b)

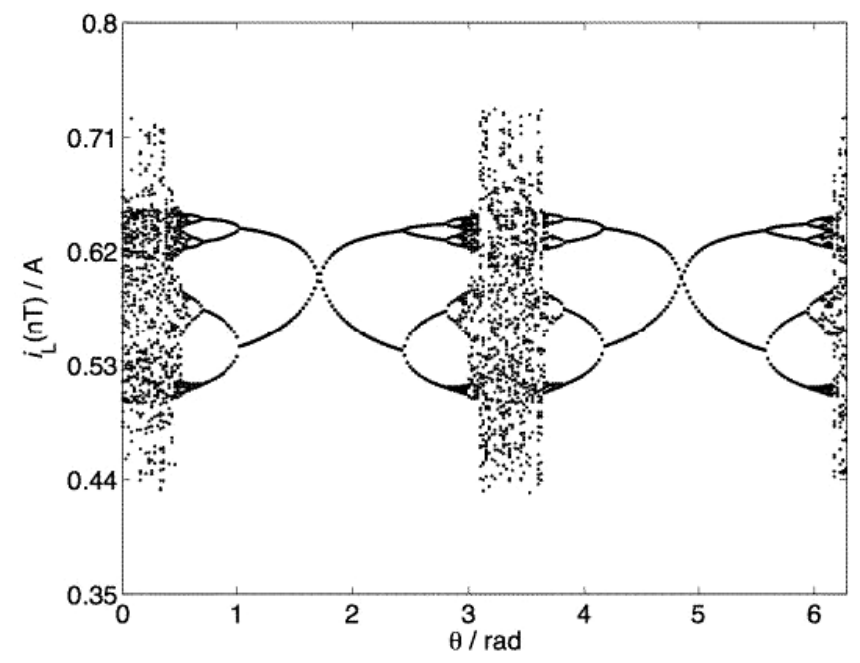

(d)

Fig. 10. Parameter-bifurcation diagram with $\theta$ as bifurcation parameter for $n f_{o}=1250 \mathrm{~Hz}$. (a) $\alpha_{v}=0.021$; (b) $\alpha_{v}=0.066$; (c) $\alpha_{v}=0.069 ;$ (d) $\alpha_{v}=0.075$. 


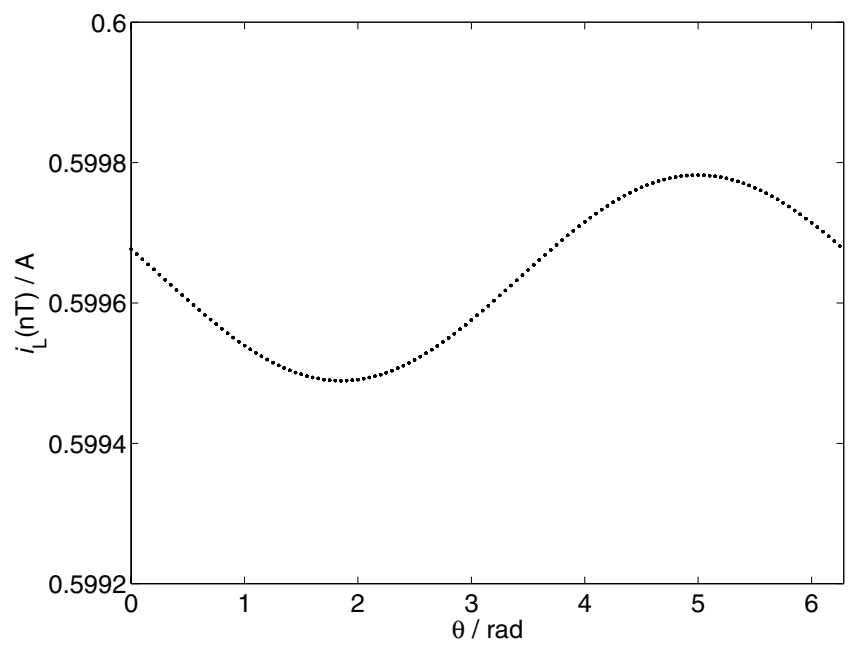

(a)

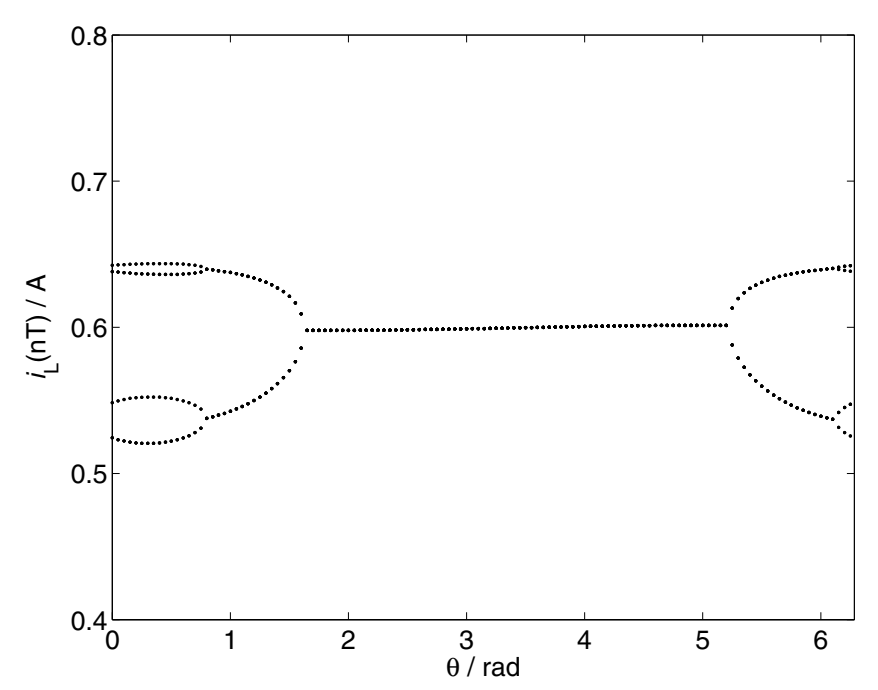

(c)

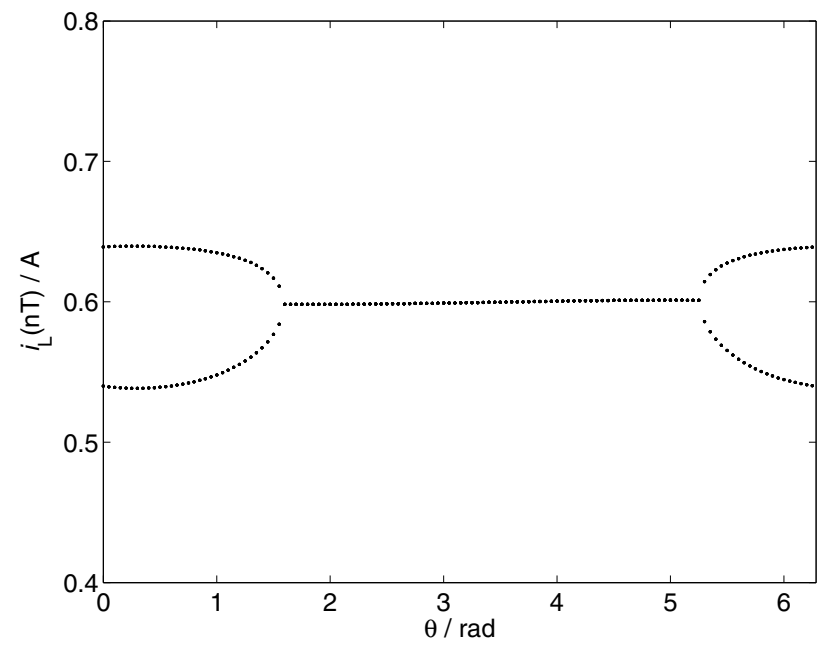

(b)

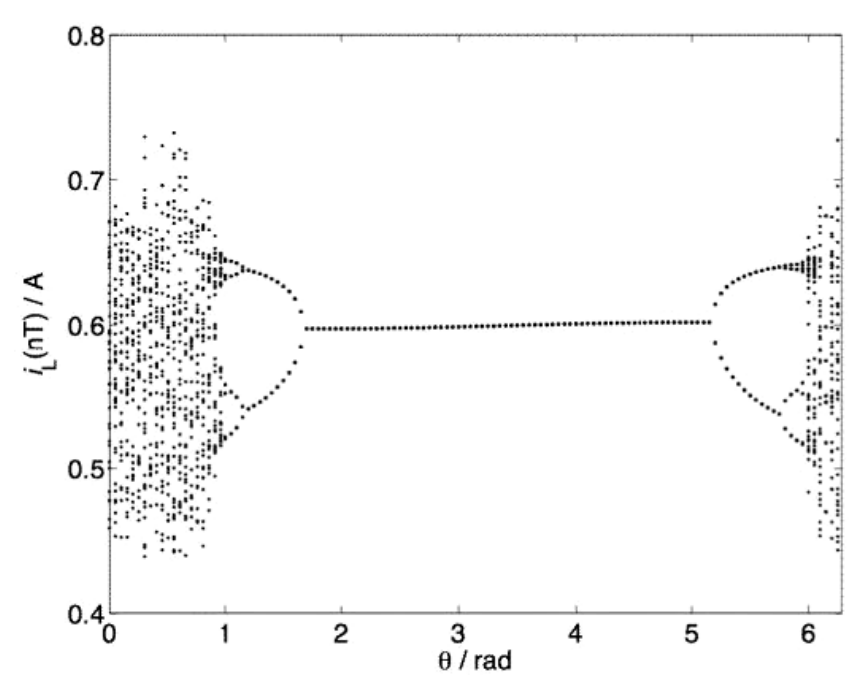

(d)

Fig. 11. Parameter-bifurcation diagrams with $\theta$ as bifurcation parameter for $n f_{o}=2500 \mathrm{~Hz}$. (a) $\alpha_{v}=0.0046$; (b) $\alpha_{v}=0.046$; (c) $\alpha_{v}=0.054 ;$ (d) $\alpha_{v}=0.069$.

is equivalent to the scenario of intermittency over $N_{\text {den }}$ intermittent period. Using the same set of interference parameters as those used in Figs. 3 to 5 , we obtain the corresponding parameter-bifurcation diagrams as shown in Figs. 10 to 12. When we compare the time-bifurcation diagrams and parameterbifurcation diagrams, we find that they look very similar and the results are consistent.

\section{Analysis of Intermittent Bifurcation}

In this section, we try to analyze the stability of the converter. After we apply the transformation, we are able to carry out the standard parameterbifurcation analysis, and the results can be used to explain the corresponding time-bifurcation of intermittency. Essentially the parameter-bifurcation over the interval of $\theta$ is exactly the same as the time-bifurcation over an intermittent period shown in Figs. 3 to 5. In this section, we will first derive the iterative discrete-time map, and then analyze the stability of the converter by computing the Jacobian [Alligood et al., 1996] and examining the loci of the characteristic multipliers [di Bernardo \& Vasca, 2000; Hamill et al., 1992; Zhou et al., 2003].

By inspecting the time-bifurcation diagram (or the corresponding parameter-bifurcation diagram), 


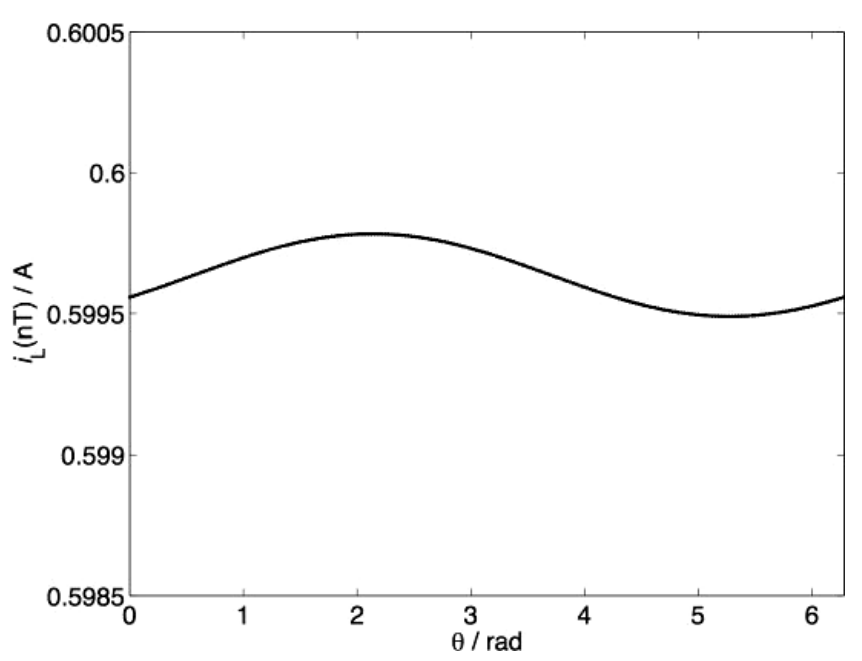

(a)

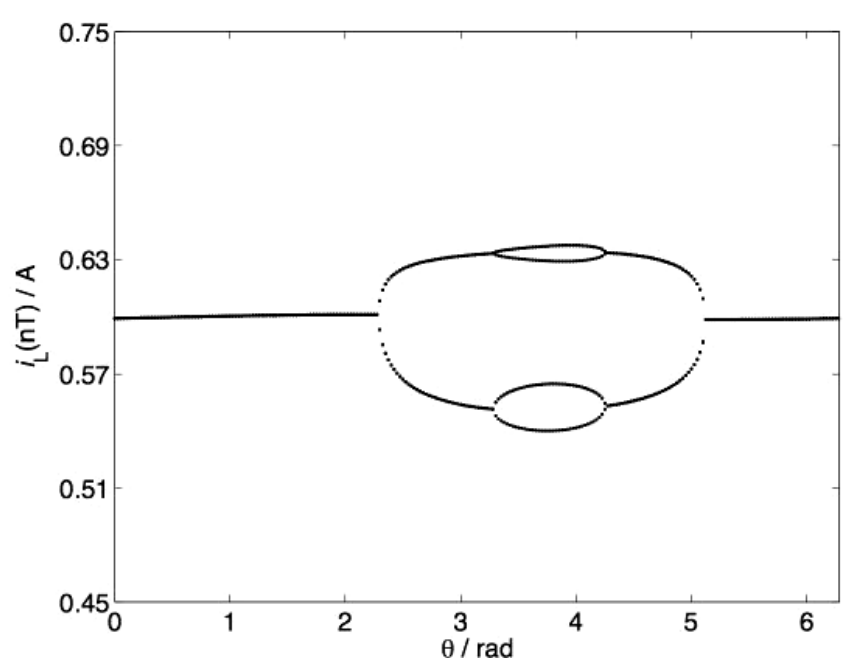

(c)

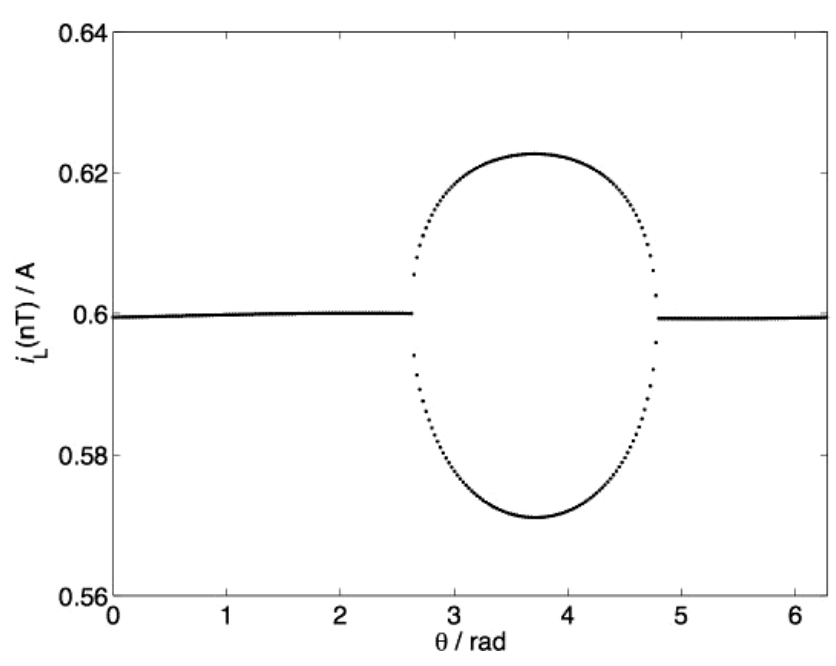

(b)

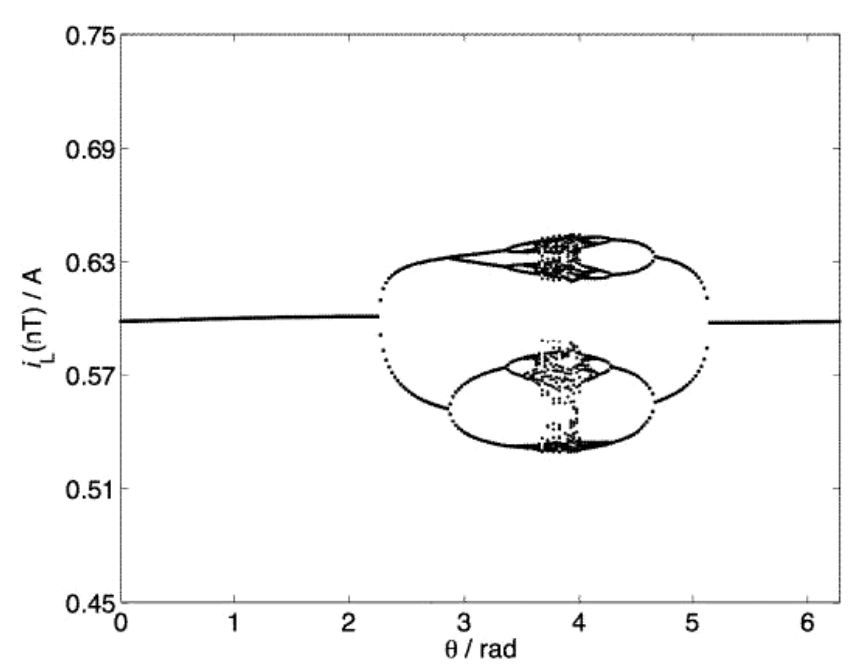

(d)

Fig. 12. Parameter-bifurcation diagrams with $\theta$ as bifurcation parameter for $n f_{o}=5000 \mathrm{~Hz}$. (a) $\alpha_{v}=0.0046$; (b) $\alpha_{v}=0.012$; (c) $\alpha_{v}=0.041 ;(\mathrm{d}) \alpha_{v}=0.053$.

it is found that the intermittency starts each intermittent period with the expected steady-state operation given by the one shown in Sec. 3. In our previous examples, there are two different cases, i.e. with the expected steady-state operation under the particular choice of parameters being a period-1 orbit for $n=1,2$, and being a period-2 subharmonic operation for $n=1 / 2$. Therefore, we will carry out the analysis for these two cases separately.

\section{1. $\alpha_{f}=n+\hat{f} / f_{o}$, where $n=1,2$}

In the case of $n$ being 1 or 2 , with the expected steady-state operation of the buck converter being a period-1 orbit, the converter will first lose stability and bifurcate from regular period- 1 to period- 2 subharmonic operation as $\theta$ increases. (Note that varying $\theta$ is equivalent to varying $t$, from (11), for a given period $1 / \hat{f}$.) A discrete-time iterative map $x_{n+1}=f\left(x_{n}\right)$ should be constructed for the analysis of stability. From the steady-state waveforms of the converter shown in Fig. 13, we can divide the operation in one period $\left(t_{n}-t_{n+1}\right)$ into two phases according to the switch state of switch $G$, as illustrated below:

(1) Phase $1, t_{n}-t_{m}$ : The state of the converter is $x_{n}$ in the beginning of this phase. In this phase, $G$ is switched off with $V_{\text {con }}>V_{\text {ramp. The state }}$ of the converter at the end of this phase, $x_{m}$, can be expressed as

$$
x_{m}=N_{\text {off }}\left(\bar{d}_{n}\right) x_{n}+M_{\text {off }}\left(\bar{d}_{n}\right) E .
$$



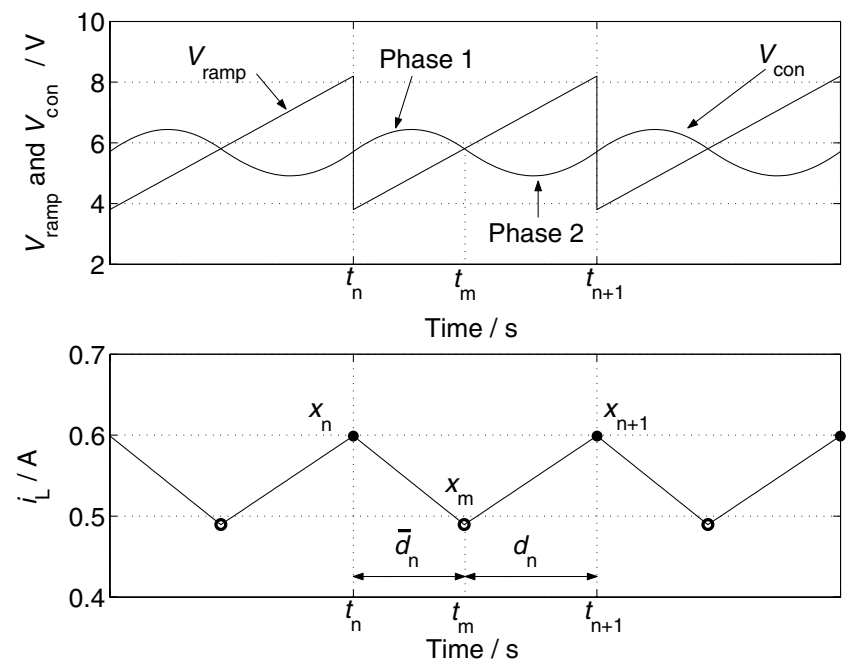

Fig. 13. Typical waveforms with $n=1,2$. Note that $G$ is switched off with $V_{\text {con }}>V_{\text {ramp }}$; and is switched on with $V_{\text {con }}<V_{\text {ramp. }}$

(2) Phase 2, $t_{m}-t_{n+1}: G$ is switched on with $V_{\text {con }}<$ $V_{\text {ramp }}$ after $t_{m}$. At the end of this phase, the state of the converter will be $x_{n+1}$, which can be expressed as

$$
x_{n+1}=N_{\text {on }}\left(1-\bar{d}_{n}\right) x_{m}+M_{\text {on }}\left(1-\bar{d}_{n}\right) E
$$

Combining (12) and (13), an iterative map over one switching period can be written as

$$
\begin{aligned}
x_{n+1}= & f\left(x_{n}, \bar{d}_{n}\right) \\
= & N_{\text {on }}\left(1-\bar{d}_{n}\right) N_{\text {off }}\left(\bar{d}_{n}\right) x_{n} \\
& +\left[N_{\text {on }}\left(1-\bar{d}_{n}\right) M_{\text {off }}\left(\bar{d}_{n}\right)\right. \\
& \left.+M_{\text {on }}\left(1-\bar{d}_{n}\right)\right] E
\end{aligned}
$$

where $d_{n}$ is the duty cycle,

$$
\begin{aligned}
\bar{d}_{n} & =1-d_{n}, \\
N_{\text {on }}(\bar{d}) & =e^{A_{\text {on }} \bar{d} T}, \\
M_{\text {on }}(\bar{d}) & =A_{\text {on }}^{-1}\left[N_{\text {on }}(\bar{d})-\mathbf{1}\right] B_{\text {on }} \\
N_{\text {off }}(\bar{d}) & =e^{A_{\text {off }} \bar{d} T}, \\
M_{\text {off }}(\bar{d}) & =A_{\text {off }}^{-1}\left[N_{\text {off }}(\bar{d})-\mathbf{1}\right] B_{\text {off }} .
\end{aligned}
$$

To complete the discrete-time model, we need to derive the defining function for the duty cycle. Essentially, we wish to find the connection between the switching instant $t_{m}$, or more precisely $\bar{d}_{n}$, and the state variables. By inspecting Fig. 13, we may define a switching function $S(\cdot)$ as

$$
S\left(\bar{d}_{n}\right)=V_{\text {con }}^{*}\left(\bar{d}_{n} T\right)-V_{\text {ramp }}\left(\bar{d}_{n} T\right),
$$

from which $G$ is switched on when $S\left(\bar{d}_{n}\right)<0$, and off otherwise. Also, $S\left(\bar{d}_{n}\right)=0$ defines the switching instants. Taking into account the perturbed control voltage and expanding (15) gives:

$$
\begin{aligned}
S\left(\bar{d}_{n}\right)= & {\left[\begin{array}{ll}
A & 0
\end{array}\right]\left[\begin{array}{c}
v_{o}\left(\bar{d}_{n} T\right)-V_{\text {ref }} \\
i_{L}\left(\bar{d}_{n} T\right)
\end{array}\right]+v_{s}\left(\bar{d}_{n} T\right) } \\
& -V_{L}-\left(V_{U}-V_{L}\right) \bar{d}_{n} T \\
= & {\left[\begin{array}{ll}
A & 0
\end{array}\right]\left[N_{\text {off }}\left(\bar{d}_{n}\right) x_{n}+M_{\text {off }}\left(\bar{d}_{n}\right) E\right]-A V_{\text {ref }} } \\
& +\alpha_{v} V_{\text {con }} \sin \left(2 \pi n \bar{d}_{n}+\theta\right)-V_{L} \\
& -\left(V_{U}-V_{L}\right) \bar{d}_{n} T
\end{aligned}
$$

The discrete-time iterative map derived above can be used to study the stability of the system. Specifically, by computing the characteristic multipliers about the equilibrium point, we can analyze the way the system loses stability as a certain parameter is varied.

First, we denote the Jacobian of the discretetime iterative map by $\Gamma$, which can be evaluated by exploiting the implicit function derivation theorem to (14) and (16), i.e.

$$
\Gamma\left(x_{n}, \bar{d}_{n}\right)=\frac{\partial x_{n+1}}{\partial x_{n}}=\frac{\partial f}{\partial x_{n}}-\frac{\partial f}{\partial \bar{d}_{n}}\left(\frac{\partial S}{\partial \bar{d}_{n}}\right)^{-1} \frac{\partial S}{\partial x_{n}}
$$

where

$$
\begin{aligned}
\frac{\partial f}{\partial \bar{d}_{n}}= & {\left[-A_{\text {on }} T N_{\text {on }}\left(1-\bar{d}_{n}\right) N_{\text {off }}\left(\bar{d}_{n}\right)\right.} \\
& \left.+N_{\text {on }}\left(1-\bar{d}_{n}\right) A_{\text {off }} T N_{\text {off }}\left(\bar{d}_{n}\right)\right] x_{n} \\
& +\left[-A_{\text {on }} T N_{\text {on }}\left(1-\bar{d}_{n}\right) M_{\text {off }}\left(\bar{d}_{n}\right)\right. \\
& +N_{\text {on }}\left(1-\bar{d}_{n}\right) N_{\text {off }}\left(\bar{d}_{n}\right) B_{\text {off }} T \\
& \left.-N_{\text {on }}\left(1-\bar{d}_{n}\right) B_{\text {on }} T\right] E \\
\frac{\partial f}{\partial x_{n}}= & N_{\text {on }}\left(1-\bar{d}_{n}\right) N_{\text {off }}\left(\bar{d}_{n}\right) \\
\frac{\partial S}{\partial \bar{d}_{n}}= & {\left[\begin{array}{ll}
A & 0
\end{array}\right] N_{\text {off }}\left(\bar{d}_{n}\right)\left(A_{\text {off }} x_{n}+B_{\text {off }} E\right) T } \\
& +2 \pi V_{\text {con }} \alpha_{v} n \cos \left(2 \pi n \bar{d}_{n}+\theta\right)-\left(V_{U}-V_{L}\right) T \\
\frac{\partial S}{\partial x_{n}}= & {\left[\begin{array}{ll}
A & 0
\end{array}\right] N_{\text {off }}\left(\bar{d}_{n}\right) }
\end{aligned}
$$

Then the characteristic multipliers, $\lambda$, can be found by solving

$$
\operatorname{det}\left[\lambda \mathbf{1}-\Gamma\left(x_{n}, \bar{d}_{n}\right)\right]_{x_{n}=X_{Q}, \bar{d}_{n}=\bar{d}_{Q}}=0
$$

where $x_{Q}$ and $\bar{d}_{Q}$ are the equilibrium values, and $\mathbf{1}$ is the identity matrix. 

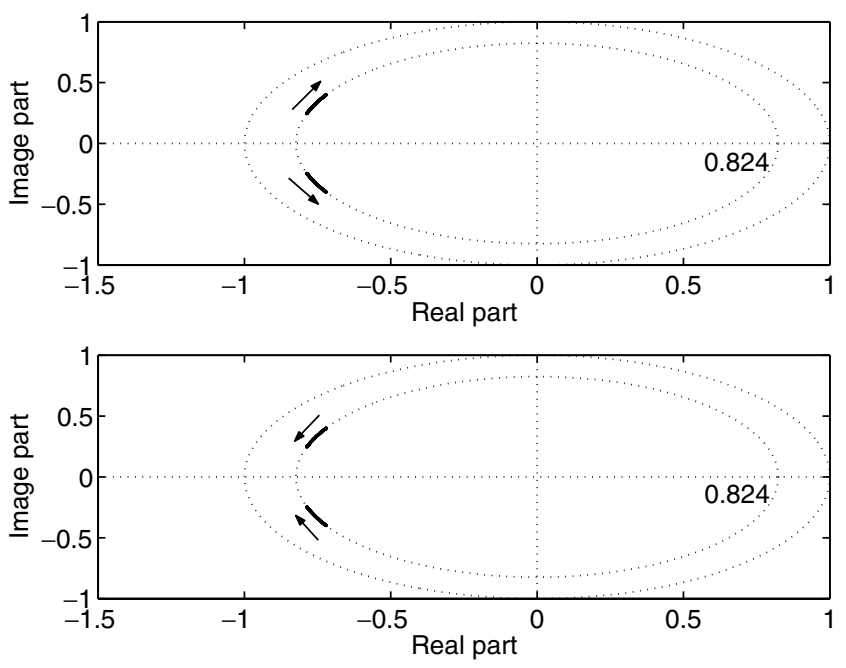

(a)
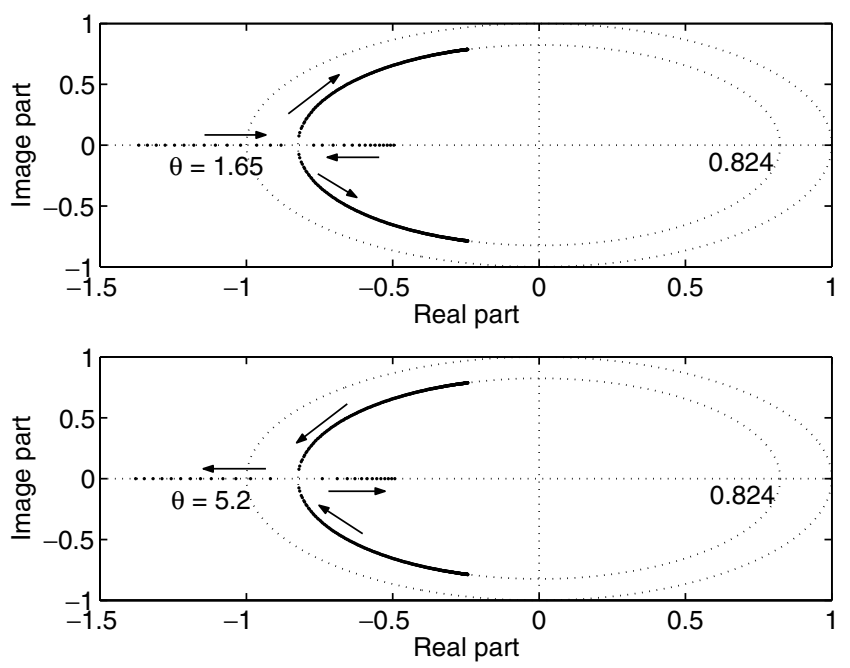

(c)
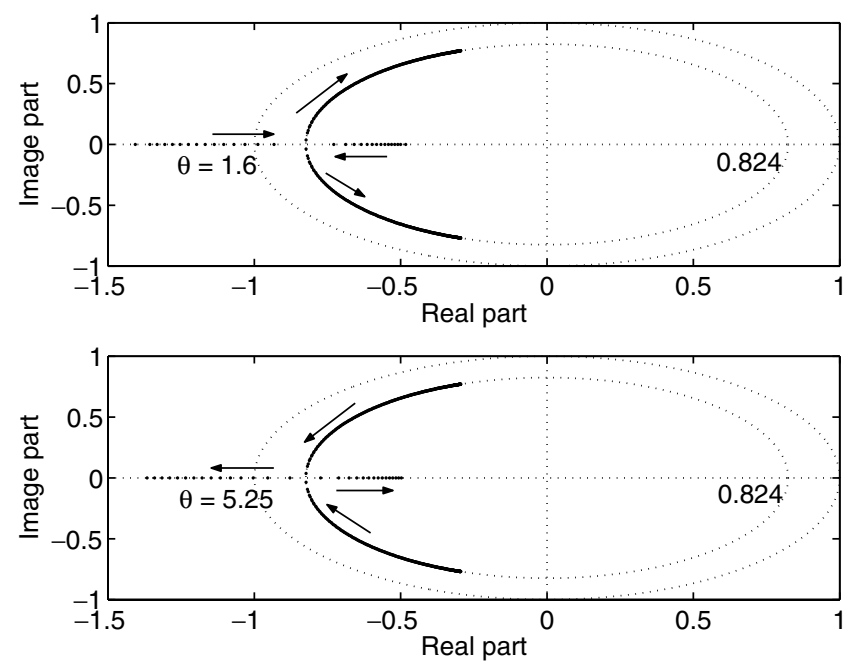

(b)
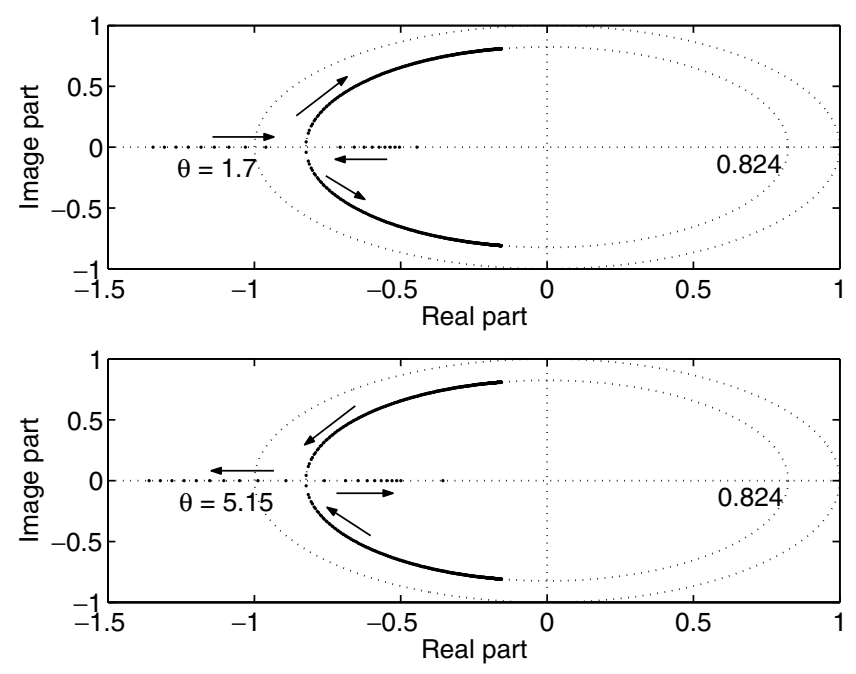

(d)

Fig. 14. Loci of the characteristic multipliers for $n f_{o}=2500 \mathrm{~Hz}$. (a) $\alpha_{v}=0.0046$; (b) $\alpha_{v}=0.046$; (c) $\alpha_{v}=0.054$; (d) $\alpha_{v}=$ 0.069 .

We now examine the movement of the characteristic multipliers as the phase shift $\theta$ varies. The loci of the characteristic multipliers are shown graphically in Figs. 14 and 15. It is noted that we use the same strength and frequency of interference as in Sec. 5. Thus, the system's stability near the equilibrium point can be studied. The movements of the loci of the characteristic multipliers agree perfectly well with the parameter-bifurcation diagrams shown in Figs. 11 and 12. We can summarize the results as follows.

(1) With very weak interference $\left(\alpha_{v}=0.0046\right.$ for $n f_{o}=2500 \mathrm{~Hz}$ and $5000 \mathrm{~Hz}$ ), the loci of the characteristic multipliers are found as in
Figs. 14(a) and 15(a). From these figures, we observe that as the phase shift $\theta$ increases from 0 , two characteristic multipliers move apart along a circle of radius less than 1 (equals 0.824 here) for the case of $n f_{o}=2500 \mathrm{~Hz}$ (for the case of $n f_{o}=5000 \mathrm{~Hz}$, two characteristic multipliers move apart along the circle of radius less than 1 in opposite directions). When $\theta$ increases further, they move back along the original path. For all values of $\theta$, they stay within the unit circle, it implies that the converter maintains its expected operation. This result is consistent with the parameter-bifurcation diagrams in Figs. 11(a) and 12(a) and the time-bifurcation diagrams in Figs. 4(a) and 5(a). 

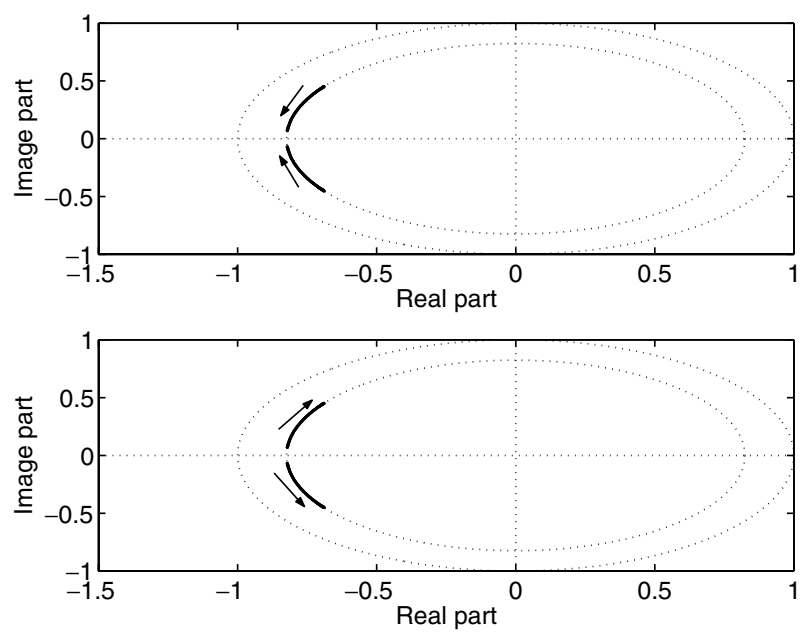

(a)
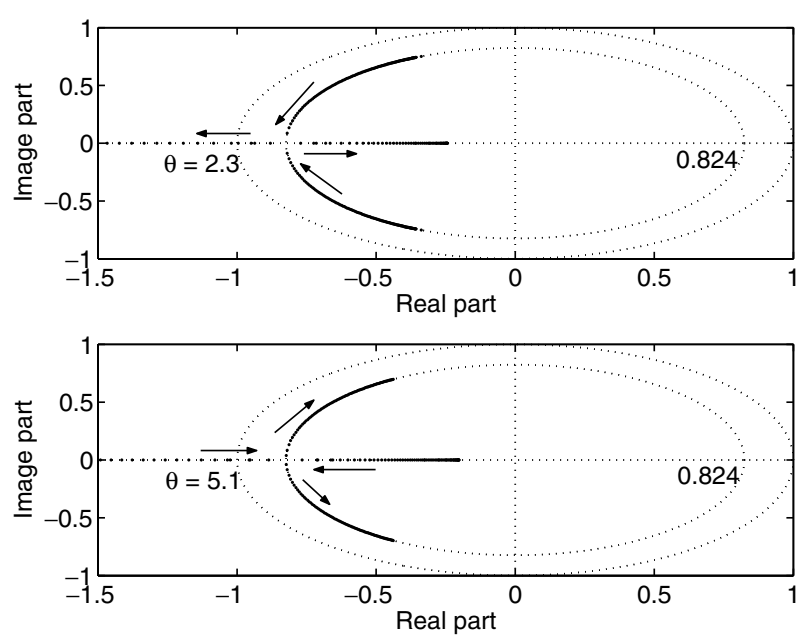

(c)
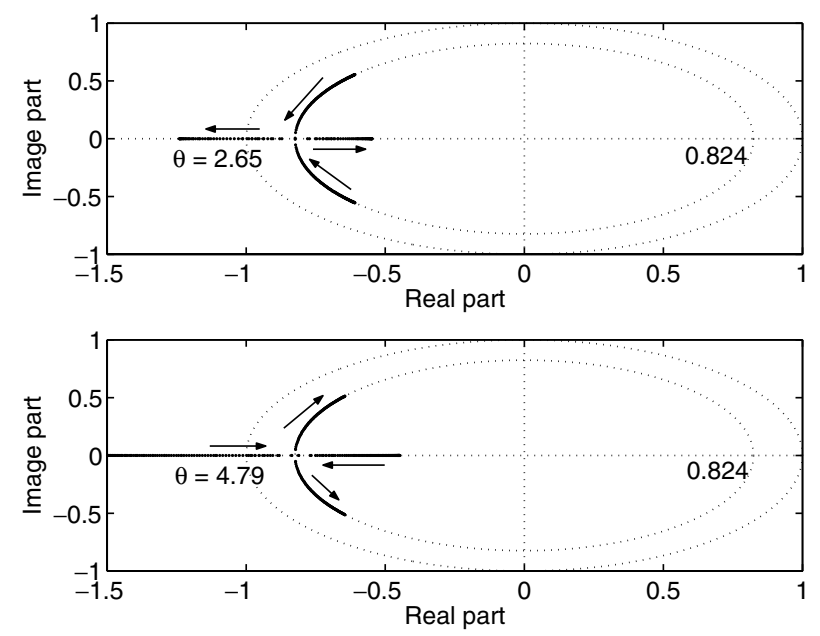

(b)
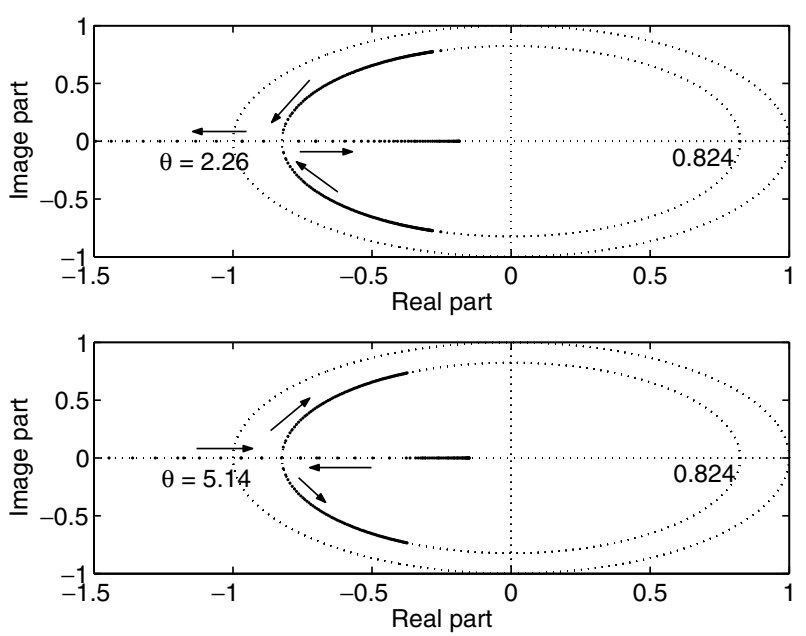

(d)

Fig. 15. Loci of the characteristic multipliers for $n f_{o}=5000 \mathrm{~Hz}$. (a) $\alpha_{v}=0.0046$; (b) $\alpha_{v}=0.012 ;$ (c) $\alpha_{v}=0.041 ;$ (d) $\alpha_{v}=$ 0.053 .

(2) As the strength of the interference increases $\left(\alpha_{v}=0.046\right.$ for $n f_{o}=2500 \mathrm{~Hz}$, and $\alpha_{v}=0.012$ for $n f_{o}=5000 \mathrm{~Hz}$ ), bifurcation is observed as $\theta$ varies, as shown in Figs. 11(b) and 12(b), which corresponds to the intermittent subharmonics in Figs. 4(b) and 5(b). This scenario can be explained in terms of the movement of the loci of the characteristic multipliers, as illustrated in Figs. 14(b) and 15(b). For the case of $n f_{o}=2500 \mathrm{~Hz}$, one characteristic multiplier crosses the unit circle along the real axis from outside when $\theta$ increases up to 1.6. Thus, the converter becomes stable as $\theta$ increases up to about 1.6. It remains in this stable operation state till $\theta=5.25$. At this point, one characteristic multiplier leaves the unit circle and the converter undergoes a period-doubling.
While for the case of $n f_{o}=5000 \mathrm{~Hz}$, one characteristic multiplier crosses the unit circle along the real axis from inside when $\theta$ increases up to 2.65 . Thus, the converter experiences a perioddoubling bifurcation and becomes unstable as $\theta$ increases up to about 2.65. It remains in this unstable operation state till $\theta=4.79$. At this point, one characteristic multiplier enters the unit circle and the converter returns to its intended regular operation.

(3) For higher values of $\alpha_{v}$ (for both $n f_{o}=2500 \mathrm{~Hz}$ and $5000 \mathrm{~Hz}$ ), the converter period-doubles up to period- 4 subharmonics and chaotic regime. The loci of the characteristic multipliers in Figs. 14(c), 15(c), 14(d) and 15(d) also locate the onset of the period-doubling bifurcation clearly. The movements of the characteristic 
multipliers are very similar to those in the previous case $\left(\alpha_{v}=0.046\right.$ for $n f_{o}=2500 \mathrm{~Hz}$, and $\alpha_{v}=0.012$ for $n f_{o}=5000 \mathrm{~Hz}$ ), and they are not repeated here.

\section{2. $\alpha_{f}=n+\hat{f} / f_{o}$, where $n=1 / 2$}

In the case of $n$ being $1 / 2$, with the expected steady-state operation of the converter being a period-2 subharmonic orbit, the converter will first lose stability and bifurcate from a regular period-2 operation to a period- 4 operation. A discrete-time iterative map in the form of $x_{n+2}=f\left(x_{n}\right)$ should be constructed for the analysis of stability. From the steady-state waveforms of the converter shown in Fig. 16, we can divide the operation over two periods $\left(t_{n}-t_{n+2}\right)$ into four phases according to the switch state of switch $G$, as illustrated below:

(1) Phase $1, t_{n}-t_{m 1}$ : The state of the converter is $x_{n}$ at the beginning of this phase. In this phase, $G$ is switched off with $V_{\text {con }}>V_{\text {ramp }}$. The state of the converter at the end of this phase, $x_{m 1}$, can be expressed as

$$
x_{m 1}=N_{\text {off }}\left(\bar{d}_{1 n}\right) x_{n}+M_{\text {off }}\left(\bar{d}_{1 n}\right) E
$$

(2) Phase 2, $t_{m 1}-t_{n+1}: G$ is switched on with $V_{\text {con }}<V_{\text {ramp }}$ after $t_{m 1}$. At the end of this phase, the state of the converter will be $x_{n+1}$, which can be expressed as

$$
\begin{aligned}
x_{n+1}= & N_{\text {on }}\left(1-\bar{d}_{1 n}\right) x_{m 1} \\
& +M_{\text {on }}\left(1-\bar{d}_{1 n}\right) E
\end{aligned}
$$
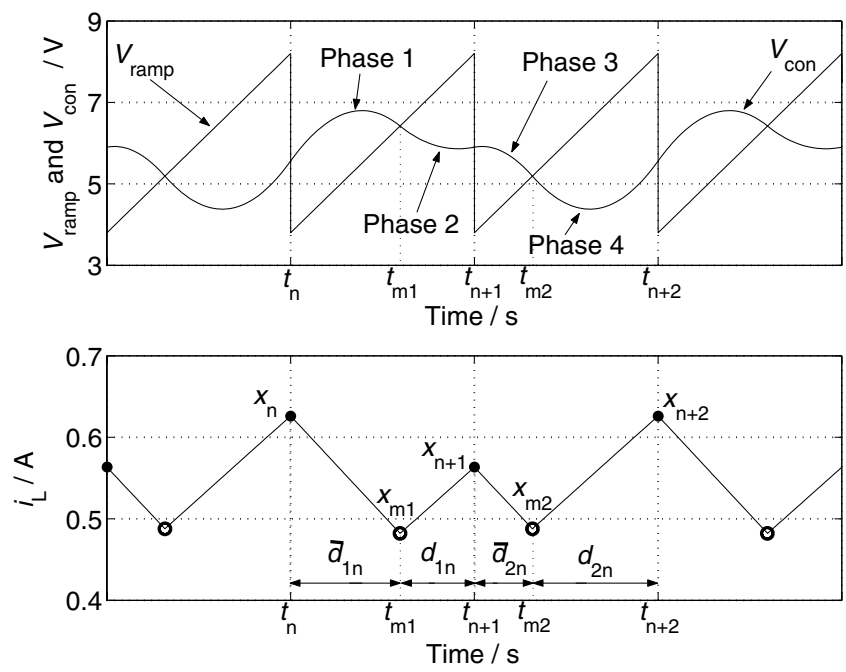

Fig. 16. Typical waveforms with $n=1 / 2$. Note that $G$ is switched off with $V_{\text {con }}>V_{\text {ramp; }}$ and is switched on with $V_{\text {con }}<V_{\text {ramp }}$.
(3) Phase $3, t_{n}+1-t_{m 2}$ : $G$ is switched off with $V_{\text {con }}>V_{\text {ramp. }}$ The state of the converter at the end of this phase, $x_{m 2}$, can be expressed as

$$
x_{m 2}=N_{\text {off }}\left(\bar{d}_{2 n}\right) x_{n+1}+M_{\text {off }}\left(\bar{d}_{2 n}\right) E
$$

(4) Phase $4, t_{m 2}-t_{n+2}: G$ is switched on with $V_{\text {con }}<V_{\text {ramp }}$ after $t_{m 2}$. At the end of this phase, the state of the converter will be $x_{n+2}$, which can be expressed as

$$
x_{n+2}=N_{\text {on }}\left(1-\bar{d}_{2 n}\right) x_{m 2}+M_{\text {on }}\left(1-\bar{d}_{2 n}\right) E
$$

Following similar procedures, we can write an iterative map over two switching periods, using (19) to $(22)$, as

$$
\begin{aligned}
x_{n+2}= & f\left(x_{n}, \bar{d}_{2 n}, \bar{d}_{1 n}\right) \\
= & N_{\text {on }}\left(1-\bar{d}_{2 n}\right) N_{\text {off }}\left(\bar{d}_{2 n}\right) N_{\text {on }}\left(1-\bar{d}_{1 n}\right) \\
& \times N_{\text {off }}\left(\bar{d}_{1 n}\right) x_{n} \\
& +N_{\text {on }}\left(1-\bar{d}_{2 n}\right) N_{\text {off }}\left(\bar{d}_{2 n}\right) N_{\text {off }}\left(1-\bar{d}_{1 n}\right) \\
& \times M_{\text {off }}\left(\bar{d}_{1 n}\right) E \\
& +N_{\text {on }}\left(1-\bar{d}_{2 n}\right) N_{\text {off }}\left(\bar{d}_{2 n}\right) M_{\text {on }}\left(1-\bar{d}_{1 n}\right) E \\
& +N_{\text {on }}\left(1-\bar{d}_{2 n}\right) M_{\text {off }}\left(\bar{d}_{2 n}\right) E \\
& +M_{\text {on }}\left(1-\bar{d}_{2 n}\right) E
\end{aligned}
$$

where $N$ 's and $M$ 's are defined as before.

Similarly, by inspecting Fig. 16, two switching functions defining the duty cycles can be written as

$$
\begin{aligned}
S_{1}\left(x_{n},\right. & \left.\bar{d}_{1 n}\right) \\
= & {\left[\begin{array}{ll}
A & 0
\end{array}\right]\left[N_{\text {off }}\left(\bar{d}_{1 n}\right) x_{n}+M_{\text {off }}\left(\bar{d}_{1 n}\right) E\right]-A V_{\text {ref }} } \\
& +\alpha_{v} V_{\text {con }} \sin \left(\pi \bar{d}_{1 n}+\theta\right)-V_{L} \\
& -\left(V_{U}-V_{L}\right) \bar{d}_{1 n} T \\
S_{2}\left(x_{n},\right. & \left.\bar{d}_{1 n}, \bar{d}_{2 n}\right) \\
= & {[A \quad 0]\left[N_{\text {off }}\left(\bar{d}_{2 n}\right) N_{\text {on }}\left(1-\bar{d}_{1 n}\right) N_{\text {off }}\left(\bar{d}_{1 n}\right) x_{n}\right.} \\
& +N_{\text {off }}\left(\bar{d}_{2 n}\right) N_{\text {on }}\left(1-\bar{d}_{1 n}\right) M_{\text {off }}\left(\bar{d}_{1 n}\right) E \\
& \left.+N_{\text {off }}\left(\bar{d}_{2 n}\right) M_{\text {on }}\left(1-\bar{d}_{1 n}\right) E+M_{\text {off }}\left(\bar{d}_{2 n}\right) E\right] \\
& -A V_{\text {ref }}-\alpha_{v} V_{\text {con }} \sin \left(\pi \bar{d}_{2 n}+\theta\right) \\
& -V_{L}-\left(V_{U}-V_{L}\right) \bar{d}_{2 n} T
\end{aligned}
$$

We can analyze the way the system loses stability of period-2 subharmonic operation by computing the characteristic multipliers of the discrete-time iterative map derived above near the equilibrium point. First, we denote the Jacobian of the discrete-time iterative map by $\Gamma$, which can be evaluated by applying the implicit function 
derivation theorem to (23) through (25), i.e.

$$
\Gamma=\frac{\partial f}{\partial x_{n}}-\frac{\partial f}{\partial \bar{d}_{1 n}}\left(\frac{\partial S_{1}}{\partial \bar{d}_{1 n}}\right)^{-1} \frac{\partial S_{1}}{\partial x_{n}}-\frac{\partial f}{\partial \bar{d}_{2 n}}\left(\frac{\partial S_{2}}{\partial \bar{d}_{2 n}}\right)^{-1}\left[\frac{\partial S_{2}}{\partial x_{n}}-\frac{\partial S_{2}}{\partial \bar{d}_{1 n}}\left(\frac{\partial S_{1}}{\partial \bar{d}_{1 n}}\right)^{-1} \frac{\partial S_{1}}{\partial x_{n}}\right]
$$

where

$$
\begin{aligned}
& \frac{\partial f}{\partial x_{n}}=N_{\text {on }}\left(1-\bar{d}_{2 n}\right) N_{\text {off }}\left(\bar{d}_{2 n}\right) N_{\text {on }}\left(1-\bar{d}_{1 n}\right) N_{\text {off }}\left(\bar{d}_{1 n}\right) \\
& \frac{\partial f}{\partial \bar{d}_{1 n}}=N_{\text {on }}\left(1-\bar{d}_{2 n}\right) N_{\text {off }}\left(\bar{d}_{2 n}\right) \frac{\partial N_{\text {on }}\left(1-\bar{d}_{1 n}\right)}{\partial \bar{d}_{1 n}} N_{\text {off }}\left(\bar{d}_{1 n}\right) x_{n} \\
& +N_{\text {on }}\left(1-\bar{d}_{2 n}\right) N_{\text {off }}\left(\bar{d}_{2 n}\right) N_{\text {on }}\left(1-\bar{d}_{1 n}\right) \frac{\partial N_{\text {off }}\left(\bar{d}_{1 n}\right)}{\partial \bar{d}_{1 n}} x_{n} \\
& +N_{\text {on }}\left(1-\bar{d}_{2 n}\right) N_{\text {off }}\left(\bar{d}_{2 n}\right) \frac{\partial N_{\text {on }}\left(1-\bar{d}_{1 n}\right)}{\partial \bar{d}_{1 n}} M_{\text {off }}\left(\bar{d}_{1 n}\right) E+N_{\text {on }}\left(1-\bar{d}_{2 n}\right) N_{\text {off }}\left(\bar{d}_{2 n}\right) N_{\text {on }}\left(1-\bar{d}_{1 n}\right) \\
& \times \frac{\partial M_{\mathrm{off}}\left(\bar{d}_{1 n}\right)}{\partial \bar{d}_{1 n}} E+N_{\mathrm{on}}\left(1-\bar{d}_{2 n}\right) N_{\mathrm{off}}\left(\bar{d}_{2 n}\right) \frac{\partial M_{\mathrm{on}}\left(1-\bar{d}_{1 n}\right)}{\partial \bar{d}_{1 n}} E \\
& \frac{\partial f}{\partial \bar{d}_{2 n}}=\frac{\partial N_{\text {on }}\left(1-\bar{d}_{2 n}\right)}{\partial \bar{d}_{2 n}} N_{\text {off }}\left(\bar{d}_{2 n}\right) N_{\text {on }}\left(1-\bar{d}_{1 n}\right) N_{\text {off }}\left(\bar{d}_{1 n}\right) x_{n} \\
& +N_{\text {on }}\left(1-\bar{d}_{2 n}\right) \frac{\partial N_{\text {off }}\left(\bar{d}_{2 n}\right)}{\partial \bar{d}_{2 n}} N_{\text {on }}\left(1-\bar{d}_{1 n}\right) N_{\text {off }}\left(\bar{d}_{1 n}\right) x_{n} \\
& +\frac{\partial N_{\mathrm{on}}\left(1-\bar{d}_{2 n}\right)}{\partial \bar{d}_{2 n}} N_{\text {off }}\left(\bar{d}_{2 n}\right) N_{\text {on }}\left(1-\bar{d}_{1 n}\right) M_{\text {off }}\left(\bar{d}_{1 n}\right) E \\
& +N_{\text {on }}\left(1-\bar{d}_{2 n}\right) \frac{\partial N_{\text {off }}\left(\bar{d}_{2 n}\right)}{\partial \bar{d}_{2 n}} N_{\text {on }}\left(1-\bar{d}_{1 n}\right) M_{\text {off }}\left(\bar{d}_{1 n}\right) E \\
& +\frac{\partial N_{\text {on }}\left(1-\bar{d}_{2 n}\right)}{\partial \bar{d}_{2 n}} N_{\text {off }}\left(\bar{d}_{2 n}\right) M_{\text {on }}\left(1-\bar{d}_{1 n}\right) E+N_{\text {on }}\left(1-\bar{d}_{2 n}\right) \frac{\partial N_{\text {off }}\left(\bar{d}_{2 n}\right)}{\partial \bar{d}_{2 n}} M_{\text {on }}\left(1-\bar{d}_{1 n}\right) E \\
& +\frac{\partial N_{\mathrm{on}}\left(1-\bar{d}_{2 n}\right)}{\partial \bar{d}_{2 n}} M_{\mathrm{off}}\left(\bar{d}_{2 n}\right) E+N_{\mathrm{on}}\left(1-\bar{d}_{2 n}\right) \frac{\partial M_{\mathrm{off}}\left(\bar{d}_{2 n}\right)}{\partial \bar{d}_{2 n}} E+\frac{\partial M_{\mathrm{on}}\left(1-\bar{d}_{2 n}\right)}{\partial \bar{d}_{2 n}} E \\
& \frac{\partial S_{1}}{\partial x_{n}}=\left[\begin{array}{ll}
A & 0
\end{array}\right] N_{\text {off }}\left(\bar{d}_{1 n}\right) \\
& \frac{\partial S_{1}}{\partial \bar{d}_{1 n}}=\left[\begin{array}{ll}
A & 0
\end{array}\right]\left[\frac{\partial N_{\mathrm{off}}\left(\bar{d}_{1 n}\right)}{\partial \bar{d}_{1 n}} x_{n}+\frac{\partial M_{\mathrm{off}}\left(\bar{d}_{1 n}\right)}{\partial \bar{d}_{1 n}} E\right]+\alpha_{v} V_{\mathrm{con}} \pi \cos \left(\pi \bar{d}_{1 n}+\theta\right)-\left(V_{U}-V_{L}\right) T \\
& \frac{\partial S_{2}}{\partial x_{n}}=\left[\begin{array}{ll}
A & 0
\end{array}\right] N_{\text {off }}\left(\bar{d}_{2 n}\right) N_{\text {on }}\left(1-\bar{d}_{1 n}\right) N_{\text {off }}\left(\bar{d}_{1 n}\right) \\
& \frac{\partial S_{2}}{\partial \bar{d}_{1 n}}=\left[\begin{array}{ll}
A & 0
\end{array}\right]\left[N_{\text {off }}\left(\bar{d}_{2 n}\right) \frac{\partial N_{\text {on }}\left(1-\bar{d}_{1 n}\right)}{\partial \bar{d}_{1 n}} N_{\text {off }}\left(\bar{d}_{1 n}\right) x_{n}+N_{\text {off }}\left(\bar{d}_{2 n}\right) N_{\text {on }}\left(1-\bar{d}_{1 n}\right) \frac{\partial N_{\text {off }}\left(\bar{d}_{1 n}\right)}{\partial \bar{d}_{1 n}} x_{n}\right. \\
& +N_{\text {off }}\left(\bar{d}_{2 n}\right) \frac{\partial N_{\text {on }}\left(1-\bar{d}_{1 n}\right)}{\partial \bar{d}_{1 n}} M_{\text {off }}\left(\bar{d}_{1 n}\right) E+N_{\text {off }}\left(\bar{d}_{2 n}\right) N_{\text {on }}\left(1-\bar{d}_{1 n}\right) \frac{\partial M_{\text {off }}\left(\bar{d}_{1 n}\right)}{\partial \bar{d}_{1 n}} E \\
& \left.+N_{\text {off }}\left(\bar{d}_{2 n}\right) \frac{\partial M_{\mathrm{on}}\left(1-\bar{d}_{1 n}\right)}{\partial \bar{d}_{1 n}} E\right]
\end{aligned}
$$




$$
\begin{aligned}
\frac{\partial S_{2}}{\partial \bar{d}_{2 n}}= & {\left[\begin{array}{ll}
A & 0
\end{array}\right]\left[\frac{\partial N_{\text {off }}\left(\bar{d}_{2 n}\right)}{\partial \bar{d}_{2 n}} N_{\text {on }}\left(1-\bar{d}_{1 n}\right) N_{\text {off }}\left(\bar{d}_{1 n}\right) x_{n}+\frac{\partial N_{\text {off }}\left(\bar{d}_{2 n}\right)}{\partial \bar{d}_{2 n}} N_{\text {on }}\left(1-\bar{d}_{1 n}\right) M_{\text {off }}\left(\bar{d}_{1 n}\right) E\right.} \\
& \left.+\frac{\partial N_{\text {off }}\left(\bar{d}_{2 n}\right)}{\partial \bar{d}_{2 n}} M_{\text {on }}\left(1-\bar{d}_{1 n}\right) E+\frac{\partial M_{\text {off }}\left(\bar{d}_{2 n}\right)}{\partial \bar{d}_{2 n}} E\right]-\alpha_{v} V_{\text {con }} \pi \cos \left(\pi \bar{d}_{2 n}+\theta\right)-\left(V_{U}-V_{L}\right) T
\end{aligned}
$$

Then, the characteristic multipliers, $\lambda$, can be found by solving

$$
\operatorname{det}\left[\lambda \mathbf{1}-\Gamma\left(x_{n}, \bar{d}_{1 n}, \bar{d}_{2 n}\right)\right]_{x_{n}=X_{Q}, \bar{d}_{1 n}=\bar{d}_{1, Q}, \bar{d}_{2 n}=\bar{d}_{2, Q}}=0
$$

where $x_{Q}, \bar{d}_{1, Q}$ and $\bar{d}_{2, Q}$ are the equilibrium values, and $\mathbf{1}$ is the identity matrix. We should note that the intermittent period is $1 / 2$ with $T_{\mathrm{int}}=$ $1 /\left(N_{\mathrm{den}} \hat{f}\right)$ as indicated in Sec. 4. Therefore, the parameter-bifurcation diagram of $\theta$ over interval
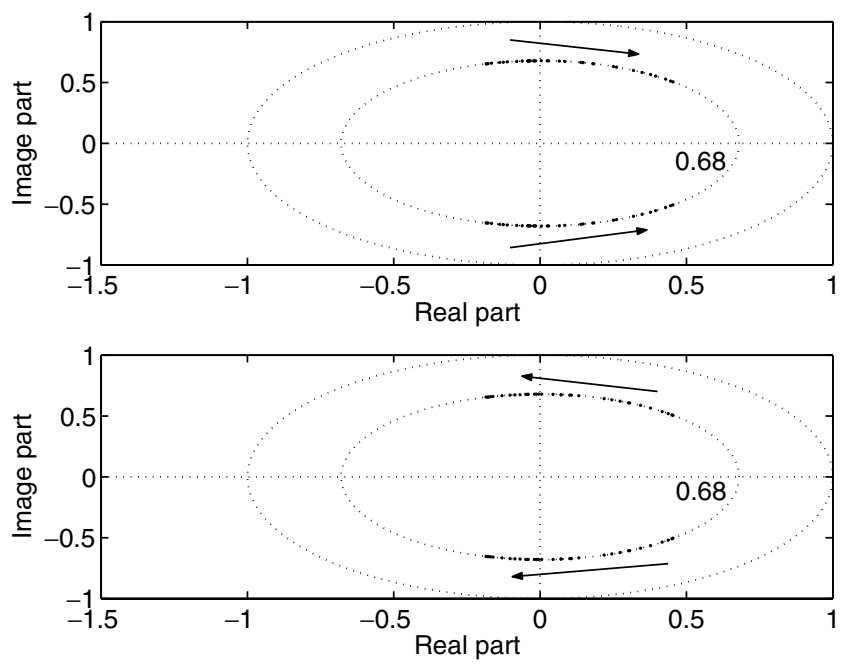

(a)
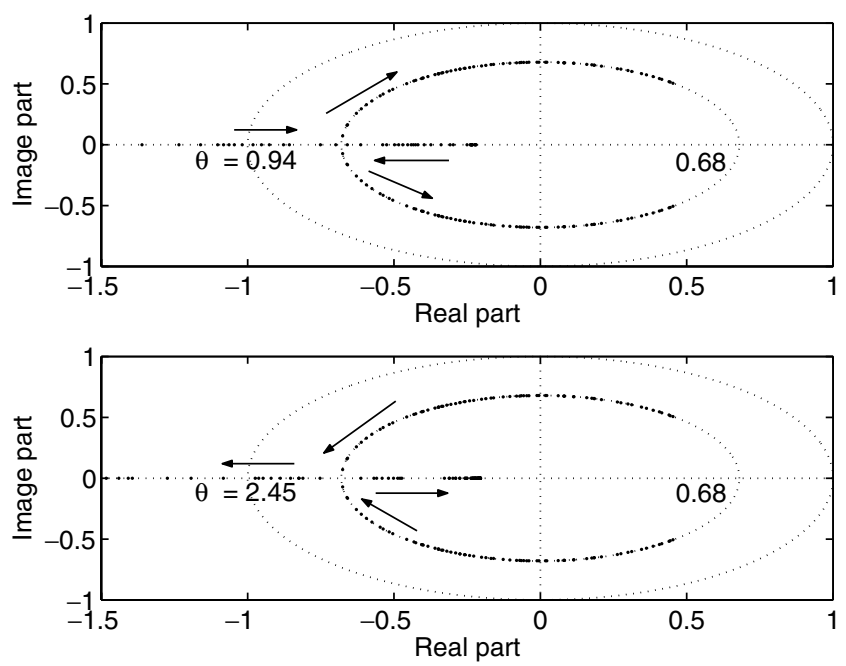

(c)
$[0, \pi]$ is exactly equivalent to that of $\theta$ over interval $[\pi, 2 \pi]$. Also, we only need to compute the characteristic multipliers with $\theta$ over interval $[0, \pi]$, and the characteristic multipliers corresponding to $\theta$ over interval $[\pi, 2 \pi]$ are the same.
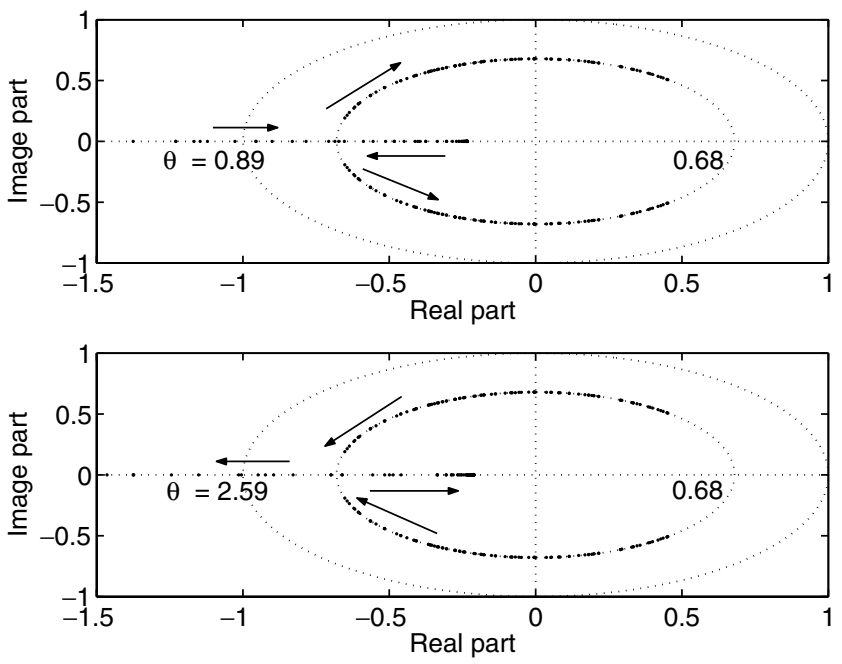

(b)
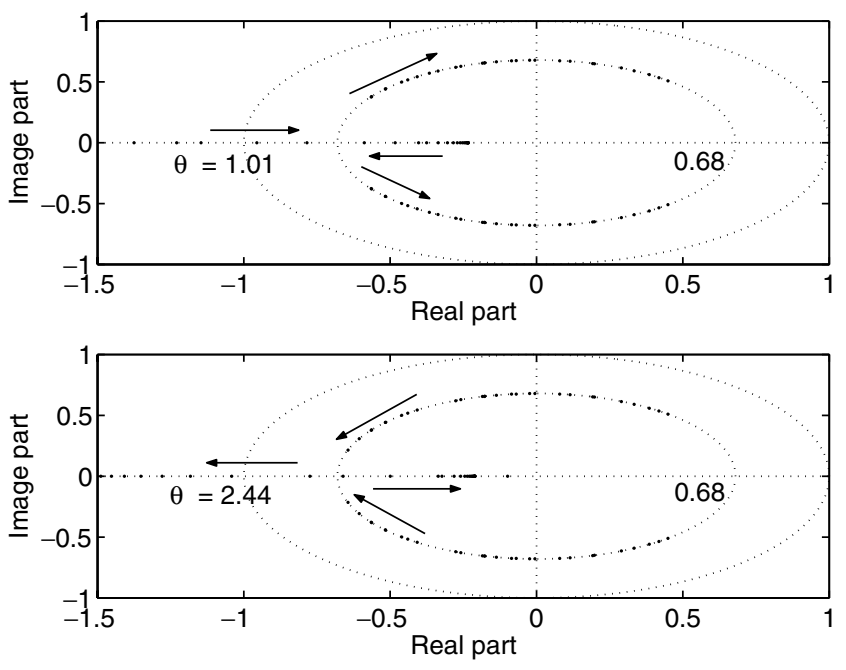

(d)

Fig. 17. Loci of the characteristic multipliers for $n f_{o}=1250 \mathrm{~Hz}$. (a) $\alpha_{v}=0.021$; (b) $\alpha_{v}=0.066$; (c) $\alpha_{v}=0.069$; (d) $\alpha_{v}=$ 0.075 . 
We now examine the movement of the characteristic multipliers as the phase shift $\theta$ varies for different values of the strength and the frequency of interference. The loci of the characteristic multipliers are shown graphically in Fig. 17. Thus, the stability of the expected steady-state operation (period-2 subharmonics) can be studied. The movements of the loci of characteristic multipliers are in perfect agreement with the parameter-bifurcation diagrams shown in Fig. 10. We can summarize the results as follows.

(1) For very weak interference $\left(\alpha_{v}=0.021\right)$, the loci of the characteristic multipliers are shown in Fig. 17(a). We observe that as phase shift $\theta$ increases from 0 , the two characteristic multipliers move along a circle of radius less than 1 (equals 0.68 here). When $\theta$ increases further, they move back along the original path. For all values of $\theta$, the corresponding characteristic multipliers are located within the unit circle, it implies that the buck converter maintains its expected steady-state operation. This result is consistent with the parameter-bifurcation diagram in Fig. 10(a) and time-bifurcation diagram in Fig. 3(a).

(2) As the strength of the interference increases $\left(\alpha_{v}=0.066\right)$, bifurcation occurs as $\theta$ is varied as shown in Fig. 10(b), which corresponds to the intermittent subharmonics of the same parameters in Fig. 3(b). This scenario can be explained in terms of the movement of the loci of the characteristic multipliers, as illustrated in Fig. 17(b). From the figure, one characteristic multiplier crosses the unit circle along the real axis from outside when $\theta$ increases up to 0.89 . Thus, the converter is stabilized to the expected steady-state period2 operation as $\theta$ increases up to about 0.89 . It remains in this stable operation state till $\theta=2.59$. At this point, one characteristic multiplier leaves the unit circle and the converter period-doubles.

(3) For higher values of $\alpha_{v}$, the converter perioddoubles up to period- 8 subharmonics or chaotic regime. The loci of the characteristic multipliers in Figs. 17(c) and 17(d) also locate the onset of the period-doubling bifurcation clearly. The movements of the characteristic multipliers are very similar to the previous case $\left(\alpha_{v}=0.066\right)$, and they are not repeated here.

\section{Conclusion}

Switching power converters are nonlinear systems which have been shown to exhibit a range of complex behavior. In this paper we attempt to explore a commonly observed but rarely explained nonlinear phenomenon in power supplies. Sources of periodic interference are easily coupled to the converters via unintended paths. We find that when the interference frequency approaches the switching frequency or its rational multiples, intermittency occurs with the intended period- $N_{\text {den }}$ operation being interrupted. We also show that the signal strength and frequency of the interference signal determine the type and period of intermittency. Furthermore, a mapping that transforms the timebifurcation to parameter-bifurcation is introduced. By applying the transformation, the intermittency can be analyzed indirectly by using the traditional numerical analysis procedures. We derive the iterative discrete-time map and examine the movement of the corresponding loci of the characteristic multipliers to explain the observed intermittency. The same line of analysis can be used to study the intermittency in other types of converters, as well as other periodically driven nonlinear systems.

\section{Acknowledgments}

This work was supported by National Natural Science Foundation of China (No. 60672023), ARC Discovery Projects Grant (No. DP0559109), and Hong Kong Polytechnic University Research Grant (No. 1BB-ZA).

\section{References}

Alligood, K. T., Sauer, T. D. \& Yorke, J. A. [1996] Chaos: An Introduction to Dynamical Systems (SrpingerVerlag, NY).

Banerjee, S. \& Verghese, G. (eds.) [2000] Nonlinear Phenomena in Power Electronics: Attractors, Bifurcations, Chaos and Nonlinear Control (IEEE Press, NY).

di Bernardo, M. \& Vasca, F. [2000] "Discrete-time maps for the analysis of bifurcations and chaos in dc/dc converters," IEEE Trans. Circuits Syst.-I 47, 130-143.

Ferreira, J. A., Willcock, P. R. \& Holm, S. R. [1997] "Sources, paths and traps of conducted EMI in switch mode circuits," Proc. IEEE Ind. Appl. Conf., pp. 1584-1591.

Fossas, E. \& Olivar, G. [1996] "Study of chaos in the buck converter," IEEE Trans. Circuits Syst.-I 43, 13-25. 
Hamill, D. C., Deane, J. H. B. \& Jefferies, D. J. [1992] "Modeling of chaotic dc-dc converters by iterated nonlinear mappings," IEEE Trans. Power Electron. 7, $25-36$

Hu, G., Xiao, J. H. \& Zhang, Z. G. [2000] Controlling Chaos (Press of Shanghai Education of Science and Technology, Shanghai) (in Chinese).

Qu, Z., Hu, G., Yang, G. \& Qin, G. [1995] "Phase effect in taming nonautonomous chaos by weak harmonic perturbations," Phys. Rev. Lett. 74, 1736-1739.

Tse, C. K. [1994] "Chaos from a buck switching regulator operating in discontinuous mode," Int. J. Circuit Th. Appl. 22, 263-278.

Tse, C. K. \& di Bernardo, M. [2002] "Complex behavior in switching power converters," Proc. IEEE 90, 768-781.

Tse, C. K., Zhou, Y., Lau, F. C. M. \& Qiu, S. S. [2003] "Intermittent chaos in switching power supplies due to unintended coupling of spurious signals," IEEE Power Electronics Specialists Conf. Record, pp. 642647.

William, T. [1994] EMC for Product Designers in Magnetism (Butterworth-Heinemann Limited, Oxford).

Wong, S. C., Tse, C. K. \& Tam, K. C. [2004] "Intermittent chaotic operation in switching power converter," Int. J. Bifurcation and Chaos 14, 2971-2978.

Yang, J., Qu, Z. \& Hu, G. [1996] "Duffing equation with two periodic forcings: The phase effect," Phys. Rev. E 53, 4402-4413.

Zhou, Y., Tse, C. K., Qiu, S. S. \& Lau, F. C. M. [2003] "Applying resonant parametric perturbation to control chaos in the buck dc/dc converter with phase shift and frequency mismatch considerations," Int. J. Bifurcation and Chaos 13, 3459-3472. 